\title{
Massive donors in interacting binaries: effect of metallicity
}

\author{
Jakub Klencki ${ }^{1}$, Gijs Nelemans ${ }^{1,2,3}$, Alina G. Istrate ${ }^{1}$, and Onno Pols ${ }^{1}$ \\ 1 Department of Astrophysics/IMAPP, Radboud University, P O Box 9010, NL-6500 GL Nijmegen, The Netherlands \\ e-mail: j.klencki@astro.ru.nl \\ 2 Institute of Astronomy, KU Leuven, Celestijnenlaan 200D, B-3001 Leuven, Belgium \\ 3 SRON, Netherlands Institute for Space Research, Sorbonnelaan 2, NL-3584 CA Utrecht, The Netherlands \\ Received Sep 12, 2018; accepted ???
}

\begin{abstract}
Metallicity is known to significantly affect the radial expansion of a massive star: the lower the metallicity, the more compact the star, especially during its post-main sequence evolution. Our goal is to study this effect in the context of binary evolution.

Using the stellar-evolution code MESA, we computed evolutionary tracks of massive stars at six different metallicities between $1.0 Z_{\odot}$ and $0.01 Z_{\odot}$. We explored variations of factors known to affect the radial expansion of massive stars (e.g., semiconvection, overshooting, or rotation). Using observational constraints, we find support for an evolution in which already at a metallicity $Z \approx$ $0.2 Z_{\odot}$ massive stars remain relatively compact $\left(\sim 100 R_{\odot}\right.$ ) during the Hertzprung-gap $(\mathrm{HG})$ phase and most of their expansion occurs during core-helium burning $(\mathrm{CHeB})$.

Consequently, we show that metallicity has a strong influence on the type of mass transfer evolution in binary systems. At solar metallicity, a case-B mass transfer is initiated shortly after the end of the main sequence, and a giant donor is almost always a rapidly expanding HG star. However, at lower metallicity, the parameter space for mass transfer from a more evolved, slowly expanding $\mathrm{CHeB}$ star increases dramatically. This means that envelope stripping and formation of helium stars in low-metallicity environments occurs later in the evolution of the donor, implying a shorter duration of the Wolf-Rayet phase (even by an order of magnitude) and higher final core masses. This metallicity effect is independent of the effect of metallicity-dependent stellar winds.

At metallicities $Z \leq 0.04 Z_{\odot}$, a significant fraction of massive stars in binaries with periods longer than 100 days engages in the first episode of mass transfer very late into their evolution, when they already have a well-developed CO core. The remaining lifetime $\left(\leqslant 10^{4} \mathrm{yr}\right.$ ) is unlikely to be long enough to strip the entire H-rich envelope. Cases of unstable mass transfer leading to a merger would produce $\mathrm{CO}$ cores that spin fast at the moment of collapse.

We find that the parameter space for mass transfer from massive donors ( $>40 M_{\odot}$ ) with outer convective envelopes is extremely small or even nonexistent. We briefly discuss this finding in the context of the formation of binary black hole mergers.
\end{abstract}

Key words. stars: massive - stars: binaries: general - stars: evolution - stars: Wolf-Rayet - gravitational waves - stars: supergiants

\section{Introduction}

Massive stars $\left(>8 M_{\odot}\right)$ play a vital role in the evolution of the Universe and various branches of astronomy. They provide feedback and chemical enrichment in star-forming galaxies and stellar clusters, they are responsible for supernovae and nucleosynthesis of heavy elements, and they lead to the formation of neutron stars and black holes (e.g., Langer 2012). One key factor that significantly affects the evolution and fate of massive stars is wind mass-loss (Smith 2014). In particular, the strength of linedriven winds of hot massive stars is metallicity dependent, as is indicated by observations and predicted theoretically (Puls et al. 1996; Vink et al. 2001; Puls et al. 2008). This introduces a relation between metallicity and the evolution of massive stars. As a result, it is believed that certain stellar-origin phenomena are predominantly or even exclusively associated with low-metallicity environments, for instance, massive stellar black holes (BHs, Zampieri \& Roberts 2009; Mapelli et al. 2009; Belczynski et al. 2010; Spera et al. 2015), single stellar origin long gamma ray bursts (GRBs, Yoon et al. 2006), chemically homogeneous stars and binaries (de Mink et al. 2009; de Mink \& Mandel 2016; Marchant et al. 2017), or pair-instability supernovae (Heger \& Woosley 2002; Heger et al. 2003; Farmer et al. 2019). Metallicity is likely to play a role in the formation efficiency of compact bi- nary mergers and gravitational-wave sources (e.g., Klencki et al. 2018; Giacobbo et al. 2018; Chruslinska et al. 2019)

Metallicity also influences stellar radii. On the main sequence (MS), stars at low metallicity remain more compact than their higher metallicity counterparts (the difference can be especially large for stars with masses $\gtrsim 50 M_{\odot}$; Sanyal et al. 2017). The sizes of more evolved post-MS massive stars can be influenced by their metallicity even more significantly (Brunish \& Truran 1982; Baraffe \& El Eid 1991). When stellar evolution tracks are compared across different metallicities, increasingly more massive-star models remain relatively compact during the phase of rapid Hertzprung-Gap (HG) expansion as the metallicity $(Z)$ decreases, see for example, Brott et al. (2011; models at $Z=0.0088,0.0047$, and 0.0021), Georgy et al. (2013; $Z=0.002)$, Groh et al. (2019; $Z=0.0004)$, Schootemeijer et al. (2019; $Z=0.0034)$, or Marigo et al. (2001; $Z=0$, Pop-III ). Such stars regain thermal equilibrium and start the core-helium burning phase $(\mathrm{CHeB})$ as B- or A-type blue supergiants (BSGs, $R \sim 100 R_{\odot}$ ) and typically reach the sizes of red supergiants (RSGs, $R \sim 1000 R_{\odot}$ ) only at the final stages of $\mathrm{CHeB}$ or during even more advanced evolutionary phases.

The way in which a star changes its radius during its evolution is especially important when it is a member of a binary system: any Roche-lobe overflow (RLOF) and mass-transfer phase 
is usually associated with a phase of expansion of the donor star. The vast majority of massive stars are formed in binary and higher-order systems (Sana et al. 2012; Duchêne \& Kraus 2013), in which, more often than not, they will at some point engage in RLOF (Sana et al. 2013). For this reason, it is essential to place the evolution of single massive stars into the binary perspective and to analyze the models from the point of view of such stars becoming donors in mass transfer episodes. Because of the effect of metallicity on the radial evolution of stars, two binary systems at different metallicities with otherwise identical initial parameters can enter mass transfer at very different ages, with the donor stars being at different evolutionary stages and leading to different fates. For instance, de Mink et al. (2008) pointed out that the small sizes of HG stars at very low metallicity $(Z=0.00001)$ can allow for many more cases of case $\mathrm{C}$ mass transfer when the donor stars have well-developed carbon-oxygen $(\mathrm{CO})$ cores. This evolutionary route may be required in some binary formation scenarios of long GRBs (see the discussion in Wolf \& Podsiadlowski 2007; and Sec. 5.5). Klencki \& Nelemans (2019; and in much more detail in Klencki et al. in prep.) suggested that mass transfer from a slowly expanding $\mathrm{CHeB}$ donor (typical for subsolar metallicity) can relax to a nuclear-timescale evolution instead of stripping the entire envelope of the giant donor on a much shorter thermal timescale, as is the case of HG donors (typical for solar metallicity, Kippenhahn \& Weigert 1967).

In this paper we argue that because of the effect it has on the stellar radii, metallicity can have significant implications for the evolution of massive binaries at various orbital periods and deserves a detailed study. To this end, we employ the MESA stellar evolution code (Paxton et al. 2011; 2013; 2015) to compute stellar tracks of massive stars between 10 and $80 M_{\odot}$ for six metallicities between $Z=0.017=Z_{\odot}$ and $Z=0.00017=0.01 Z_{\odot}$. We investigate several variations in the input physical parameters that are known to affect the radial expansion of massive stars (e.g., semiconvection, overshooting, and rotation) and constrain the models by observations of supergiants in the Small Magellanic Cloud (SMC) and in the Milky Way.

The paper is organized as follows. In Section 2 we describe our computational method and physical assumptions. In Section 3 we present our results: stellar evolutionary tracks, and the inferred binary parameter ranges for different cases of mass transfer. In Section 4 we compare our reference model and its variations to the observational constraints. In Section 5 we discuss the robustness of our results as well as the implications of our findings. We conclude in Section 6. A detailed study of the actual mass transfer phases is presented in a separate paper (Klencki et al. in prep.).

\section{Stellar models: physical ingredients}

We employed the MESA stellar evolution code of Paxton et al. $(2011 ; 2015 ; 2019)^{1}$. We modeled convection using the mixinglength theory (Böhm-Vitense 1958) with a mixing-length parameter $\alpha=1.5$, and we adopted the Ledoux criterion for convection. We accounted for semiconvection following Langer et al. (1983) with a relatively high efficiency parameter $\alpha_{\mathrm{SC}}=100.0$, as guided by the recent results of Schootemeijer et al. (2019). While the value of $\alpha_{\mathrm{SC}}$ has been shown to have limited effect on the MS evolution, its effect on the post-MS expansion in radius can be substantial (see Sec. 5.1 and App. B). In Sec. 3.3 we also explore variations with $\alpha_{\mathrm{SC}}=1.0$ and 10.0.

MESA version r11554, http://mesa. sourceforge. net/.
We accounted for convective overshooting above the hydrogen-burning core by applying the step overshooting formalism with an overshooting length $\sigma_{\text {ov }}$ of 0.345 pressure scale heights based on the calibration of Brott et al. (2011) for a $16 \mathrm{M}_{\odot}$ star. This also agrees with the best fits to the SMC supergiant population obtained by Schootemeijer et al. (2019) for $\sigma_{\mathrm{ov}}$ of 0.33 . While there is increasing evidence for a relation between $\sigma_{\mathrm{ov}}$ and the stellar mass (e.g., Claret \& Torres 2018; also Grin et al., private communication), a quantitative calibration for the massive stars is still lacking. We assumed the same amount of overshooting above the helium-burning core. For consistency with Schootemeijer et al. (2019), we assumed no overshooting associated with burning shells and convective zones in stellar envelopes. In Sec. 3.3 we also explore variations with the lower convective overshooting efficiency calibrated by Choi et al. (2016).

We modeled stellar winds following Brott et al. (2011). For hot $\left(T_{\text {eff }}>25 \mathrm{kK}\right)$ and hydrogen-rich $\left(X_{\mathrm{S}}>0.7\right)$ stars, we adopted the mass-loss rates from Vink et al. (2000; 2001). For hydrogen-poor stars $\left(X_{\mathrm{S}}<0.4\right)$, we applied the wind mass-loss as calculated by Hamann et al. (1995), divided by 10 (Yoon et al. 2006). For stars with intermediate hydrogen abundances $\left(0.4<X_{\mathrm{S}}<0.7\right)$, we linearly interpolated between the two. For cooler stars $\left(T_{\text {eff }}<25 \mathrm{kK}\right)$, we took the maximum of the two wind mass-loss rates as calculated from the methods of Vink et al. (2001) and Nieuwenhuijzen \& de Jager (1990). All these mass-loss rates for hydrogen-rich and hydrogen-poor stars include a metallicity dependence of $\dot{M} \propto\left(Z / Z_{\odot}\right)^{0.85}$ (Vink et al. 2001; Vink \& de Koter 2005).

For the value of solar metallicity, we assumed $Z_{\odot}=0.017$ (Grevesse \& Sauval 1998). Given a metallicity $Z$, we calculated the initial helium abundance as $Y$ as $Y=Y_{\text {proto }}+\left(Y_{\odot}-\right.$ $\left.Y_{\text {proto }}\right) Z / Z_{\odot}$, where $Y_{\odot}=0.28$ Grevesse et al. (1996) and $Y_{\text {proto }}=$ 0.249 (Planck Collaboration et al. 2016). The hydrogen abundance follows as $X=1-Y-Z$. Relative abundances of other elements were assumed to be same as in Grevesse et al. (1996).

We avoided using the MLT++ option in MESA (Paxton et al. 2013) in our reference model. While MLT++ typically helps with many numerical problems in evolving a massive star until the Wolf-Rayet (WR) stage, it also artificially reduces the stellar radii during the giant phase and therefore affects the predictions for mass transfer evolution. We consider a variation with MLT++ in Sec. 3.3 (and also App. A). We used nuclear reaction networks provided with MESA: basic.net for $\mathrm{H}$ and $\mathrm{He}$ burning, and co_burn . net for $\mathrm{C}$ and $\mathrm{O}$ burning.

We computed models in the mass range between 10 and $80 \mathrm{M}_{\odot}$ in steps of $2 M_{\odot}$ (below $20 M_{\odot}$ ) or $2.5 \mathrm{M}_{\odot}\left(\right.$ above $20 M_{\odot}$ ) at six different metallicities: $Z=0.017,0.0068,0.0034,0.0017$, 0.00068 , and 0.00017 (which correspond to $1.0 Z_{\odot}, 0.4 Z_{\odot}$, $0.2 Z_{\odot}, 0.1 Z_{\odot}, 0.04 Z_{\odot}$, and $0.01 Z_{\odot}$ ). Most of our models are nonrotating, but we also explored rotating models with the initial rotation rate set to $40 \%$ of the critical value $\left(\Omega / \Omega_{\text {crit }}=0.4\right)$. In these models we included the effects of Eddington-Sweet circulation, secular shear instabilities, and the Goldreich-SchubertFricke instability, with an efficiency factor $f_{c}=1 / 30$ (see the calibration to nitrogen enrichment in rotating stars and the references in Heger et al. 2000; Brott et al. 2011).

There is no one common termination condition for all our models. Because the goal is to study single stellar tracks in the context of binary evolution and massive interacting binaries, we are interested in the evolution of single stars roughly until they have reached their largest radius. By that point, if they were members of binary systems, they would usually have gone into RLOF. In some cases, typically at low metallicity or for the 
low-mass end of our grid, stars continue to expand even during the late evolutionary stages of advanced burning and reach their maximum sizes near the very end of their lives. In these cases we evolved our models until the onset of oxygen burning. At this stage, a massive star is only about a year away from core collapse (depending on the mass), and there is no time left for any significant change in its size. In the remaining cases, the expansion of stars is quenched earlier through strong stellar winds. When a significant fraction of the envelope mass has been lost, the evolution in the Hertzprung-Russel (HR) diagram turns around toward higher effective temperatures. From that point onward, as increasingly more mass is lost from the envelope, the star continues to decrease in radius to eventually become a WR star. This part of the evolution is not relevant in the case of interacting binaries: either a RLOF would have occurred earlier, or it is not going to occur at all. In these cases we only evolved the models until the final turnaround in the HR diagram has been reached (taking into account the possibility of blue loops). The resulting mass transfer and post-mass transfer evolution is discussed in Klencki et al. (in prep.).

The MESA inlists (input files) used in this work are available at http: //cococubed. asu.edu/mesa_market/ inlists.html. All the output files, in particular stellar tracks, can be found at https://zenodo.org/communities/mesa.

\section{Results}

\subsection{Stellar tracks and radius evolution of massive stars}

In Figure 1 we show HR diagrams with evolutionary tracks of single stars with masses between 10 and $80 \mathrm{M}_{\odot}$ computed for solar $\left(Z=Z_{\odot}=0.017\right)$, subsolar (SMC-like, $Z=0.2 Z_{\odot}=$ $0.0034)$, and very low metallicity $\left(Z=0.01 Z_{\odot}=0.00017\right)$. In the appendix (Fig. C.1) we provide additional plots for intermediate metallicity values $\left(0.4 Z_{\odot}, 0.1 Z_{\odot}\right.$, and $\left.0.04 Z_{\odot}\right)$. Only the evolution until the maximum radial expansion and the subsequent decrease in radius by $\sim 20 \%$ is shown (i.e., excluding the final leftward evolution toward WR stars in the case of highmass and high-metallicity models). Circles in Fig. 1 mark the position of a star during its post-MS evolution taken every 50,000 years. Thus, a clustering of circles corresponds to the relatively long-lived phase of core-helium burning $(\mathrm{CHeB})$. It also indicates which part of the HR diagram is most likely to be occupied by a population of observed single stars (excluding MS stars), as predicted by our models. A lack of circles corresponds to the MS evolution and to phases of rapid expansion: the HG (between the end of MS and the onset of $\mathrm{CHeB}$ ) and the helium HG (HeHG, between the end of $\mathrm{CHeB}$ and the onset of carbon burning). The track colors correspond to the central helium abundance.

When the location and evolution of $\mathrm{CHeB}$ stars in the HR diagram across different metallicities (i.e. the clustering of the circles) are compared, a metallicity trend in the post-MS radius evolution of massive stars is evident. The lower the metallicity, the smaller (and hotter) the massive stars at the end of the rapid $\mathrm{HG}$ expansion and during the subsequent $\mathrm{CHeB}$ phase. At solar metallicity (top panel), models below $\sim 65 M_{\odot}$ expand all the way to the red giant branch (RGB, $\log T_{\text {eff }} \lesssim 3.7$ ) during the short-lived $\mathrm{HG}$ phase, and almost their maximum radii at that stage $\left(R_{\mathrm{HG} \text {;max }} \sim 300-2000 R_{\odot}\right.$, depending on the mass). At this point, the models with masses $\lesssim 40 M_{\odot}$ begin the slower core-helium burning evolution, whereas the more massive models quickly turn around toward the left in the HR diagram because of extensive mass loss. In the case of even more massive models $\left(M>65 M_{\odot}\right)$, strong winds quench expansion at smaller sizes (up to $\sim 100 R_{\odot}$ ). At SMC-like metallicity (middle panel, $Z=0.2 Z_{\odot}$ ), the post-MS radius evolution of models between $\sim 16$ and $\sim 40 \mathrm{M}_{\odot}$ is significantly different to their solar metallicity counterparts. Instead of expanding all the way to the RGB during the $\mathrm{HG}$ phase, these models regain thermal equilibrium at much smaller radii $\left(\sim 100 R_{\odot}\right)$ as slowly evolving $\mathrm{CHeB}$ stars. At very low metallicity (bottom panel, $Z=0.01 Z_{\odot}$ ), the onset of the $\mathrm{CHeB}$ phase is located at even higher effective temperature (and smaller radius) for the entire mass range 10-80 $M_{\odot}$, and there is only a very small gap in the HR diagram that is due to the HG phase.

The position of the terminal-age MS (TAMS) changes with metallicity as well: the lower the metallicity, the hotter and smaller the MS stars (with the exception of the most massive models at solar metallicity). The largest differences between metallicities appear at the high-mass end of our grid ( $\left.\gtrsim 40 M_{\odot}\right)$. Notably, around the $40-50 M_{\odot}$ range at solar metallicity (top panel) and above $\sim 60 M_{\odot}$ at SMC-like metallicity (middle panel), models expand up to $\sim 1000 R_{\odot}$ at the end of the MS. This is a signature of inflated envelopes, and this phenomenon was previously described by Kato (1985) for very massive MS stars and by Petrovic et al. (2006) for WR stars. In such stars, the Eddington limit is locally exceeded sufficiently close to the top of their envelopes (in this case, at the iron opacity peak). To prevent $L_{\text {rad }}$ from exceeding $L_{\text {Edd }}$, the layers above expand substantially and the envelope becomes extremely diluted and extended in radius (i.e., inflated), with a density inversion formed at the top (see Gräfener et al. 2012; Sanyal et al. 2015; for details). The differences in position of the TAMS at the high-mass end between different metallicities are primarily associated with the relative strength of stellar winds and the metallicity dependence of the envelope inflation in massive stars (Sanyal et al. 2017). The small sizes of our $M>65 M_{\odot}$ models at solar metallicity are in general agreement with the models by Sanyal et al. (2015), whose models never reach an effective temperature of $T_{\text {eff }} \approx 15 k K(\sim 4.2$ in log $)$ above a certain mass.

In the case of very low metallicity (bottom panel, $Z=$ $0.01 Z_{\odot}$ ), most of the models reach the end of $\mathrm{CHeB}$ while still in the blue part of the HR diagram $\left(\log \left(T_{\text {eff }} / \mathrm{K}\right) \sim 4.1\right)$. These models expand significantly during the subsequent contraction of the $\mathrm{CO}$ core and the HeHG phase and reach the RGB by the very end of their evolution, sometimes shortly $(\sim 1000 \mathrm{yr})$ before the final core collapse. This is also the case for $Z=0.04 Z_{\odot}$ models in Fig. C.1, but to a lesser extent.

In summary, depending on the metallicity, a substantial expansion in radius can occur at a completely different evolutionary stage of a massive star. We illustrate this in Fig. 2, where we show the time evolution of the radius of a $25 M_{\odot}$ model for three different metallicities. At solar metallicity, most of the expansion occurs during the HG phase, when the star expands rapidly on a thermal timescale. At subsolar SMC-like metallicity $\left(Z=0.2 Z_{\odot}\right)$, most of the expansion takes place during $\mathrm{CHeB}$, when the star is in thermal equilibrium and the radius changes slowly on a nuclear timescale. Very low metallicity stars expand significantly only after the end of $\mathrm{CHeB}$ during another phase of rapid out-of-equilibrium expansion: the HeHG phase. What follows is the core-carbon burning stage (CCB).

\subsection{Binary parameter ranges for different donor types}

In this section we take a binary perspective: we use the stellar tracks presented in the previous section to determine the evolutionary state of a donor star at the onset of mass transfer depending on the binary orbital period and metallicity. In order to do 


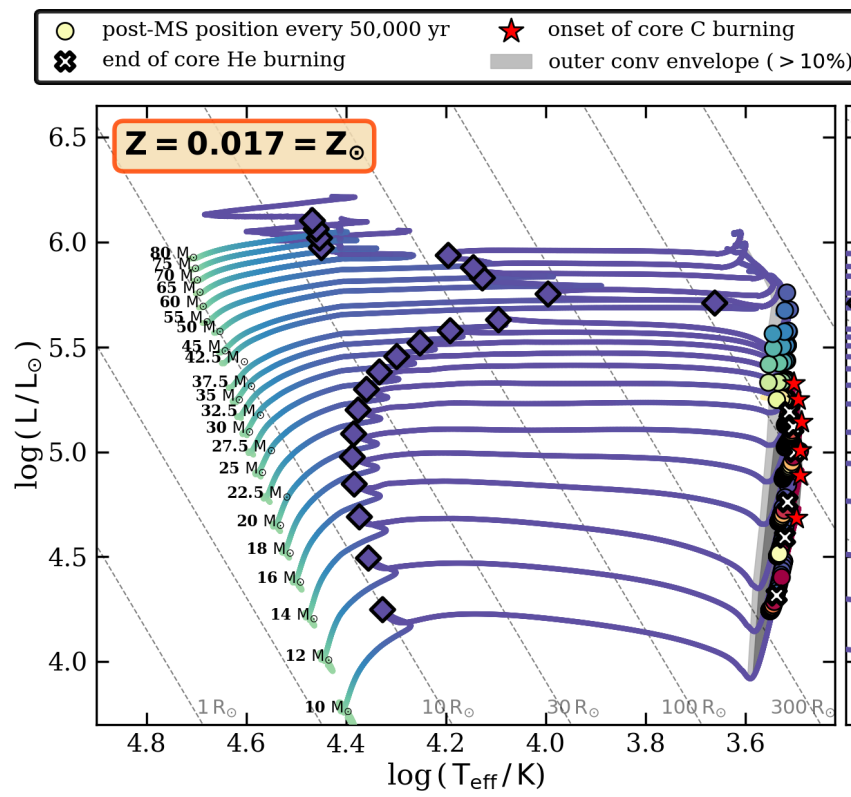

outer conv envelope $(>20 \%)$
TAMS
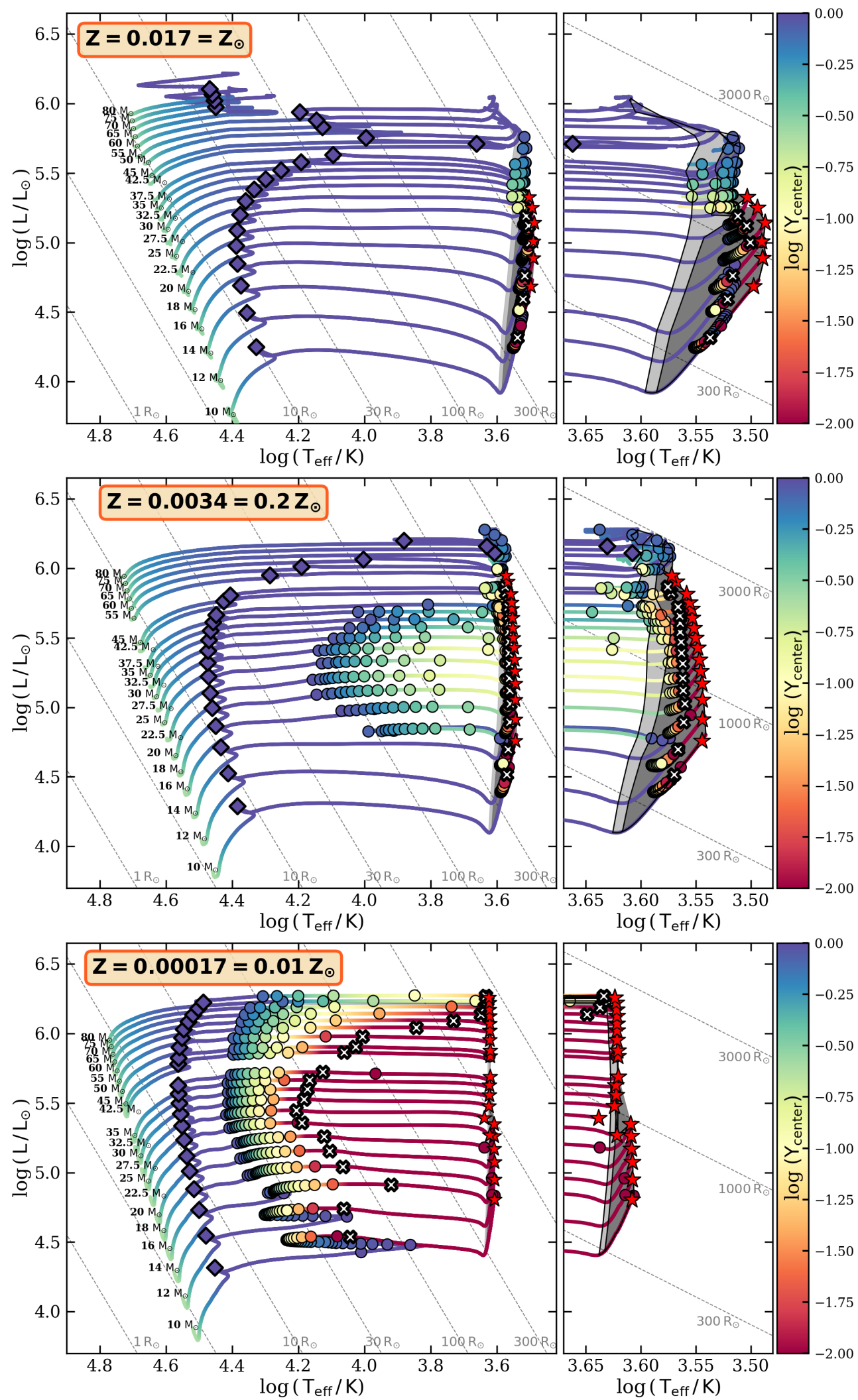

Fig. 1: Evolutionary tracks of single stars of masses between 10 and $80 \mathrm{M}_{\odot}$ computed at three different metallicities: solar (top), SMC-like (middle), and very low metallicity $Z=0.01 Z_{\odot}$ (bottom). See Fig. C. 1 for tracks at three additional metallicities. Filled circles mark the position of a star during its post-MS evolution taken every 50,000 years. Color indicates the central helium abundance $\left(Y_{\mathrm{C}}\right.$, in logarithmic scale). With a cross we mark the end of core-helium burning $\left(Y_{\mathrm{C}}<10^{-3}\right)$, and with a red star we mark the onset of core-carbon burning $\left(X_{\mathrm{C}}^{\mathrm{carbon}}<0.95 X_{\mathrm{C} ; \max }^{\text {carbon }}\right)$. Shaded regions mark stars with outer convective envelope layers of at least $10 \%$ (or 20\%) of the mass of the entire star. For tracks that turn around towards the hotter part of the HR diagram (and the WR regime) because of extensive mass loss, we only show the evolution until shortly after the maximum radius has been reached. Article number, page 4 of 19 


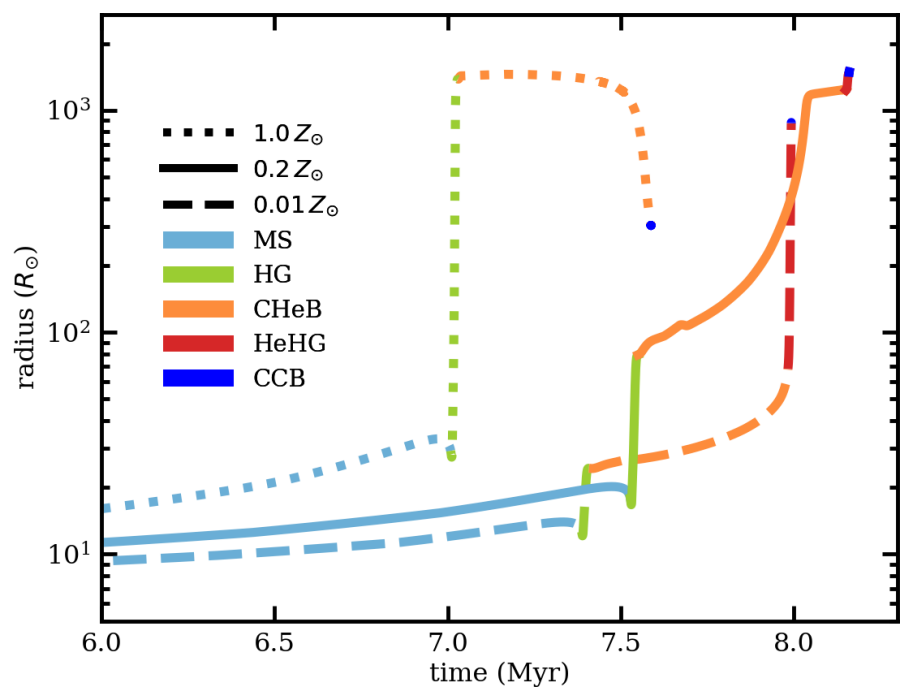

Fig. 2: Radius evolution of a $25 M_{\odot}$ single star model at solar $\left(Z=0.017=Z_{\odot}\right)$, SMC-like $\left(Z=0.0034=0.2 Z_{\odot}\right)$, and very low metallicity $\left(Z=0.00017=0.01 Z_{\odot}\right)$. The lines are colorcoded according to different evolutionary stages.

so, we assume that our single stellar models represent the evolution of the more massive component in a binary system until the eventual RLOF. We assume that the companion has half the mass of the primary star (mass ratio $q=0.5$ ) and that its mass loss in winds is negligible compared to that of the primary. For simplicity, we assume that the amount of mass accreted by the companion from the wind is negligible and that its mass is constant. We then compute the effect of the wind mass loss of the primary on the binary orbit by assuming that all the mass that the primary loses carries specific angular momentum equal to the specific orbital angular momentum of the primary.

The less massive companion can be considered to be either a less massive MS star or a stellar black hole (BH). The latter case would mean that the now more massive stellar component is what used to be the secondary star in a zero-age binary, and that the star that used to be the actual primary has already collapsed into a $\mathrm{BH}$. Before $\mathrm{BH}$ formation, the binary has most likely gone through a phase of mass transfer. In this case, the now more massive stellar component of the BH binary has previously been an accretor.

With these assumptions, we plot in Fig. 3 in different colors the evolutionary state of the massive donor star at the point of RLOF as a function of the initial donor mass (i.e., at the zero-age main sequence, ZAMS, not at RLOF) and initial orbital period for different metallicities. When the companion is assumed to be a stellar $\mathrm{BH}$, the initial period roughly corresponds to the orbital period at the moment of the $\mathrm{BH}$ formation. We neglect the effect of spin-orbit coupling by tidal interactions, which would shrink the pre-RLOF orbits by a small factor (see, e.g., Hurley et al. 2002) and shift the boundaries in Fig. 3 to somewhat higher initial orbital period values.

Several trends with metallicity are present in Fig. 3. We describe them below.

1. The lower the metallicity, the more evolved the donor stars at the point of RLOF. In particular, at $Z=Z_{\odot}$ and $0.4 Z_{\odot}$ , almost all cases of mass transfer from an evolved (postMS) donor involve a rapidly expanding HG star (green). As the metallicity decreases, however, the parameter space for RLOF initiated by a slowly expanding $\mathrm{CHeB}$ supergiant in- creases (orange) and also includes increaslingly more massive donors. This is a natural consequence of the fact that low-metallicity stars expand less strongly during the immediate post-MS expansion (HG phase) and can regain thermal equilibrium as much smaller $\mathrm{CHeB}$ stars $\left(\sim 100 R_{\odot}\right)$ than their higher metallicity counterparts $\left(\sim 1000 R_{\odot}\right)$, see Sect. 3.1. We discuss the implications of this trend for the binary formation channel of stripped helium stars in Sec. 5.3. The difference in post-MS radius expansion between lowand high -metallicity stars may also have significant consequences for the mass transfer evolution itself, see discussion in Sec. 5.4.

2. The lower the metallicity, the smaller the parameter space for RLOF from convective donors (marked with the hatched area in Fig. 3). The parameter space for convective donors disappears almost completely in the case of the more massive $\left(M>40 M_{\odot}\right)$ models at very low metallicity $\left(Z=0.04 Z_{\odot}\right.$ and $\left.Z=0.01 Z_{\odot}\right)$. It is also quenched in the case of the most massive models $\left(M>65 M_{\odot}\right)$ at solar metallicity because these models only attain relatively small sizes. Only a small fraction of massive $\mathrm{BH}$ binaries evolve through mass transfer initiated by a convective envelope donor. This fact may have important consequences for the common-envelope (CE) formation channel of double $\mathrm{BH}$ binary mergers (see also Sec. 3.4 and the discussion in Sec. 5.5).

3. The lower the metallicity, the larger the parameter space for a late RLOF, that is, RLOF from a star that is only several thousand years away from core collapse. For a metallicity between $0.017\left(Z_{\odot}\right)$ and $0.0017\left(0.1 Z_{\odot}\right)$, the parameter space for such a late RLOF is very small (even smaller than for convective donors) and limited to $M \lesssim 40 M_{\odot}$. However, for very low metallicities, that is, $Z=0.04 Z_{\odot}$ and $0.01 Z_{\odot}$, the probability of a late RLOF grows significantly, as highlighted by the cyan threshold lines. See Sec. 5.5 for a further discussion.

4. The lower the metallicity, the smaller the parameter space for mass transfer from MS donors. This is to a large extent a consequence of the formation of inflated envelopes in massive MS stars that evolve near the Eddington limit (Sanyal et al. 2015). The sets of tracks at higher metallicities $\left(1.0,0.4\right.$, and $\left.0.2 Z_{\odot}\right)$ all include a mass range in which the models become inflated and expand up to $\sim 1000 R_{\odot}$ at the end of the MS (which corresponds to initial orbital periods for RLOF of $\gtrsim 1000$ days in Fig. 3). As the metallicity decreases, this mass range shifts toward higher masses (see also Sanyal et al. 2017). As a result, for $Z \leq 0.1 Z_{\odot}$ the envelopes of MS stars do not become inflated within the mass limits of our grid (up to $80 M_{\odot}$ ). It should be noted that normal not inflated MS stars at lower metallicity are also smaller than their higher metallicity counterparts.

\subsection{Model variations}

In order to illustrate the uncertainty in binary parameter ranges for the different evolutionary states of donor stars presented in Fig. 3, we explore in Fig. 4 several variations in the input parameters for one particular metallicity: $Z=0.0017=0.1 Z_{\odot}$ (see Fig. C.2 in the Appendix for additional figures at different metallicities). Fig. 4a corresponds to the reference set of stellar tracks presented in the previous sections, computed using the assumptions described in Sec. 2. In particular, our reference model assumes step overshooting with $\sigma_{\text {ov;step }}=0.345$ (Brott et al. $2011)$, high efficiency of semiconvective mixing $\left(\alpha_{\mathrm{sc}}=100.0\right)$, no MLT++ approach, and nonrotating models. 

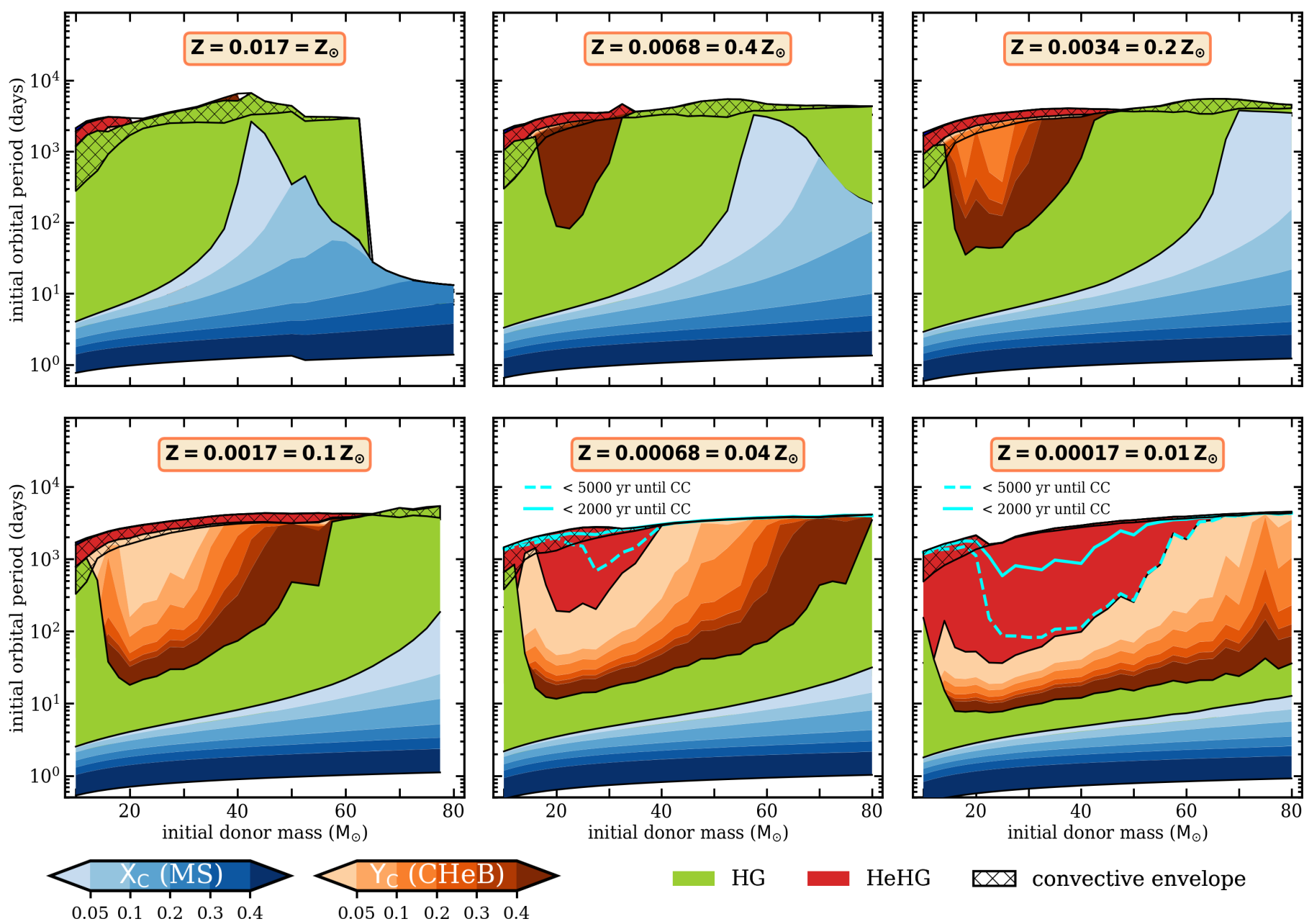

$\square \mathrm{X}$ convective envelope

Fig. 3: Evolutionary state of a donor star at the point of RLOF in massive interacting binaries as a function of initial donor mass (at the ZAMS) and initial orbital period, estimated based on the single stellar tracks presented in Sec. 3.1. The companion is assumed to be twice less massive (initial mass ratio $q=0.5$ ), and it can be viewed as either a less massive star or under additional assumptions about the previous evolution (see text) as a stellar BH. Different shades of blue (orange) correspond to different central abundances of hydrogen (helium), indicating how advanced the MS $(\mathrm{CHeB})$ evolution is. Hatched regions indicate donors with outer convective envelopes (at least 10\% in mass coordinate). Cyan lines mark the threshold above which the donors are only 2000 or 5000 years away from the core-collapse (only shown for the two lowest metallicities for clarity).

The model Fig. $4 \mathrm{~b}$ assumes a different parameterization of convective overshooting (see Sec. 3.6.2 of Choi et al. 2016), one calibrated to reproduce the shape of the MS turnoff in the open cluster M67 (Magic et al. 2010). It is expressed according to the exponential formulation of overshooting (Herwig 2000) with the characteristic length $\sigma_{\mathrm{ov} \text {; exp }}=0.016$ for the core overshooting. This value is roughly equivalent to $\sigma_{\text {ov;step }}=0.2$ in the step overshooting description. Additionally, we tested that the calibration of overshooting carried out by Brott et al. (2011) (based on the value of $\log (g)$ at the Henyey hook of $\sim 16 M_{\odot}$ stars in the LMC clusters NGC 2004 and N11) can be best fit in the exponential overshooting description with $\sigma_{\text {ov;exp }} \approx 0.03$. Thus, the Choi exponential parameterization effectively results in smaller overshooting than the step overshooting parameterization employed in Brott et al. (2011) and in our reference model.

The model in Fig. 4c assumes somewhat less efficient semiconvection $\left(\alpha_{\mathrm{sc}}=10\right)$ than in the reference model $\left(\alpha_{\mathrm{sc}}=100\right)$. Both values appear to be consistent with the tentative observational constraints (Schootemeijer et al. 2019; see also Sec. 4). In Fig. $4 \mathrm{~d}$ we show a model with even less efficient semiconvection, $\alpha_{\mathrm{sc}}=1$. This model is disfavored based on the above-mentioned observations, see also Sec.4.1.

The model in Fig. 4e includes the MLT++ approach in MESA, which was developed to model convection in radiationdominated superadiabatic envelope layers (Paxton et al. 2013). In short, the MLT++ option enforces a reduction in the temperature gradient in radiation-dominated convective zones, which effectively reduces superadiabacity and prevents the formation of density inversions. A more detailed explanation is provided in Appendix A, where we also briefly discuss a link between density inversions in envelopes of massive stars and enhanced mass-loss rates beyond the Humphreys-Davidson limit. Crucially, MLT++ also increases the effective temperature of the model and thus reduces the radius expansion of massive models, which in turn reduces the parameter space for mass transfer in massive wide systems. This is shown the bottom left panel of Fig. 4 and in the comparison of two HR diagrams of stellar tracks computed with and without MLT++ in Fig. 5. Other methods of preventing density inversions (e.g., using the density scale height or increasing the mass-loss rate) have a similar 

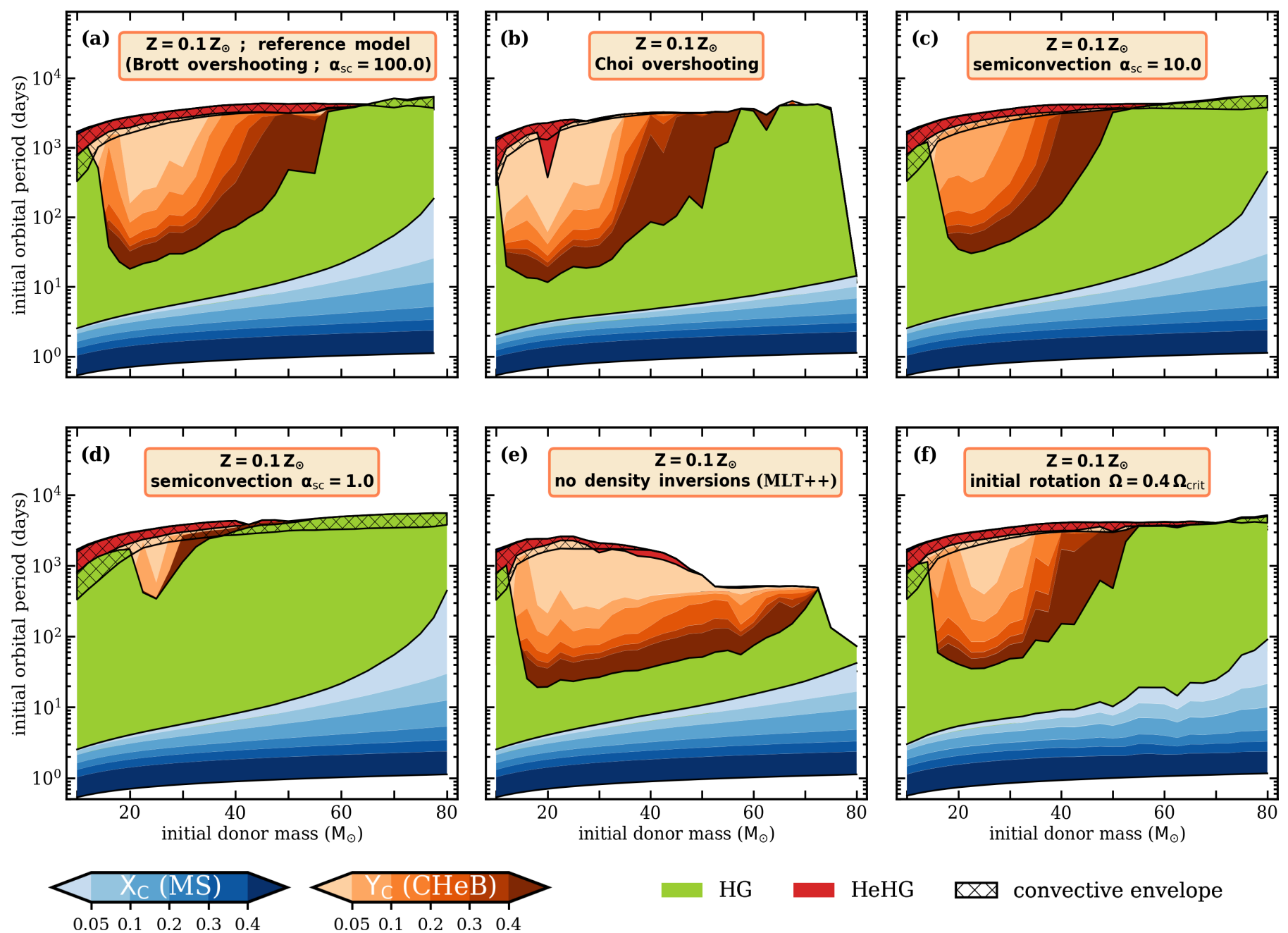

convective envelope

Fig. 4: Same as Fig. 3, but computed for one particular metallicity $\left(Z=0.0017=0.1 Z_{\odot}\right)$ and exploring several variations in the input parameters to the model with respect to the reference model presented in the previous sections (and in the top left panel): different overshooting parameterization (Choi et al. 2016), lower efficiency of semiconvective mixing $\left(\alpha_{\mathrm{sc}}=10\right.$ or $\left.\alpha_{\mathrm{sc}}=1\right)$, application of MLT++ treatment of convection in radiation-dominated superadiabatic envelope layers (Paxton et al. 2013), and initial rotation velocity $\Omega=0.4 \Omega_{\text {crit }}$. In the case of $Z=0.2 Z_{\odot}$, for which observation of evolved supergiants SMC can be used to constrain the models, none of the model variations presented above can be excluded, except for the one with $\alpha_{\mathrm{sc}}=1$; see Sec. 4 .

effect of increasing the effective temperatures of massive supergiants (e.g., Maeder \& Meynet 1987).

Finally, in Fig. 4f we show a model with evolution tracks of rotating stars with an initial angular velocity $\Omega / \Omega_{\text {crit }}=0.4$. This value was chosen to allow for a comparison with the GENEVA tracks (e.g., Georgy et al. 2013; Groh et al. 2019). Similarly to Schootemeijer et al. (2019), we find that such moderate rotation does not have a significant effect on the post-MS radius evolution. However, we note that for a more rapid initial rotation rate $\Omega / \Omega_{\text {crit }} \approx 0.6$, the most massive models in our grid at $Z=0.1 Z_{\odot}$ $\left(\gtrsim 65 M_{\odot}\right)$ would enter the chemically homogeneous evolution regime.

Similar figures to Fig. 4 for another four metallicities $(Z=$ $0.4 Z_{\odot}, 0.2 Z_{\odot}, 0.04 Z_{\odot}$, and $0.01 Z_{\odot}$ ) can be found in the Appendix in Fig. C.2. In Sec. 4 we show that for $Z=0.2 Z_{\odot}$ none of the model variations discussed above, except for the model with $\alpha_{\text {sc }}=1$, can be confidently excluded based on the observed samples of supergiants in the $\mathrm{SMC}\left(Z_{\mathrm{SMC}} \approx 0.2 Z_{\odot}\right)$. At even lower metallicities, the model uncertainties are all the more unconstrained.

\subsection{Convective envelope donors}

Whether or not a donor star has an outer convective envelope is particularly important for the question of mass transfer stability (e.g., Hjellming \& Webbink 1987; Soberman et al. 1997). In short, convective stars respond to mass loss by adiabatically expanding ${ }^{2}$, whereas stars with radiative envelopes respond by adiabatically contracting. For this reason, mass transfer from stars with outer convective envelopes is more likely to become dynamically unstable, possibly leading to a CE evolution (Webbink 1984; Hjellming \& Webbink 1987; Ivanova et al. 2013; Ge et al. 2015; Pavlovskii \& Ivanova 2015). To take this into account, population synthesis codes need to assume different stability criteria depending on whether the donor has a radiative or convective envelope. Analytical fits to evolutionary tracks that many of such codes are based on (e.g., the SSE/BSE method Hurley et al. 2000; 2002) do not usually include an explicit information

2 This may no longer be true in stars with a sufficiently well-developed superadiabatic layer in the outer envelope (Woods \& Ivanova 2011; Pavlovskii \& Ivanova 2015). 

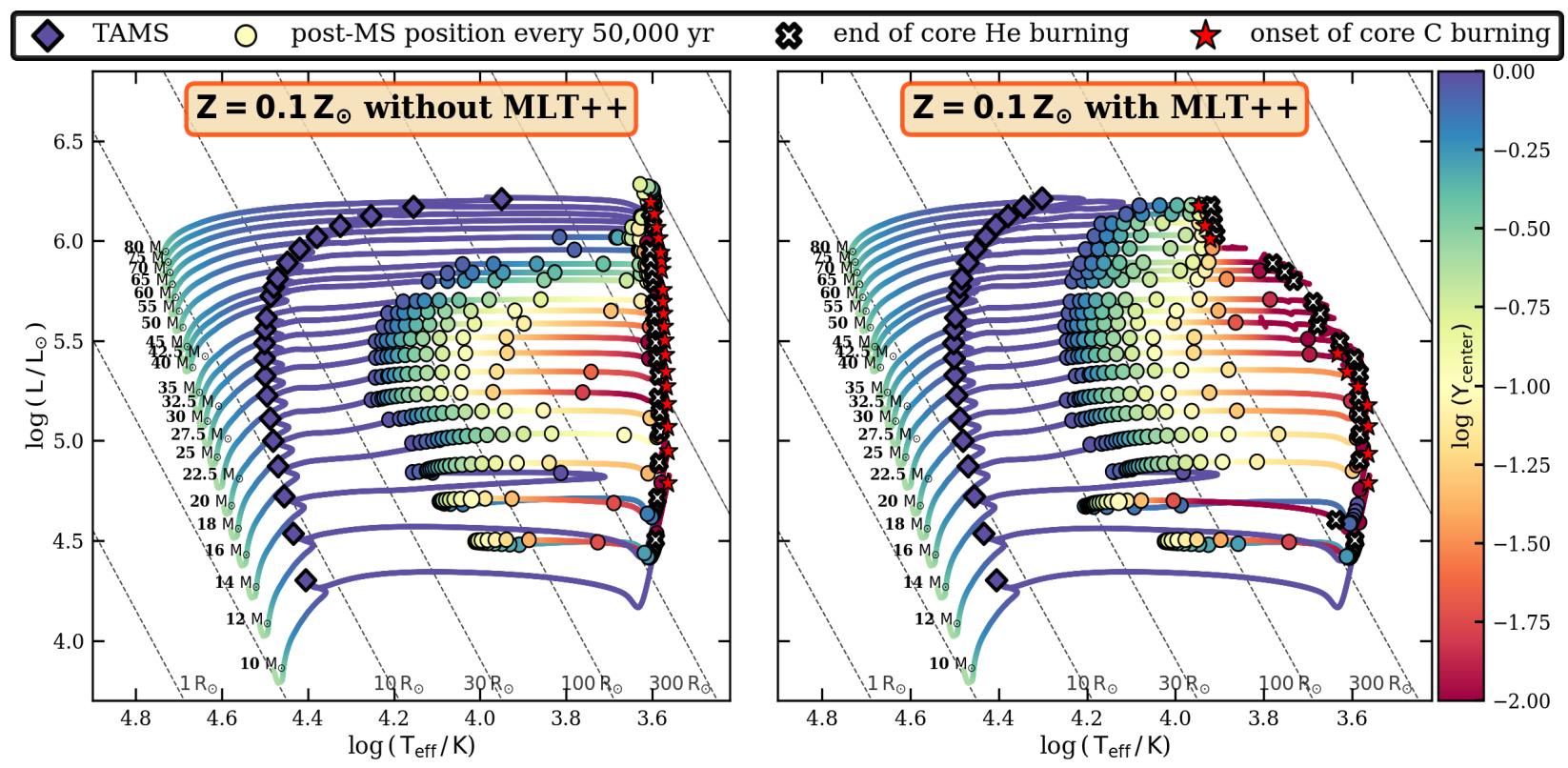

Fig. 5: Effect of MLT++ on the evolution of massive stars in the HR diagram. The various scatter points have the same meaning and color scale as in Fig. 1 and C.1. In the most massive stars, in which the density in regions with $L_{\mathrm{rad}} / L_{\mathrm{Edd}}>1$ is so small that it limits the efficiency of convection, a temperature gradient becomes superadiabatic and a density inversion develops in order to stabilize the structure (see App. A). The MLT++ approach gradually smooths the temperature gradient and reduces superadiabaticy, also removing density inversions and the $L_{\mathrm{rad}} / L_{\mathrm{Edd}}$ excess in the most massive stars. A direct consequence of the MLT++ treatment of convection is that the effective temperature becomes higher, as illustrated by the above figure.

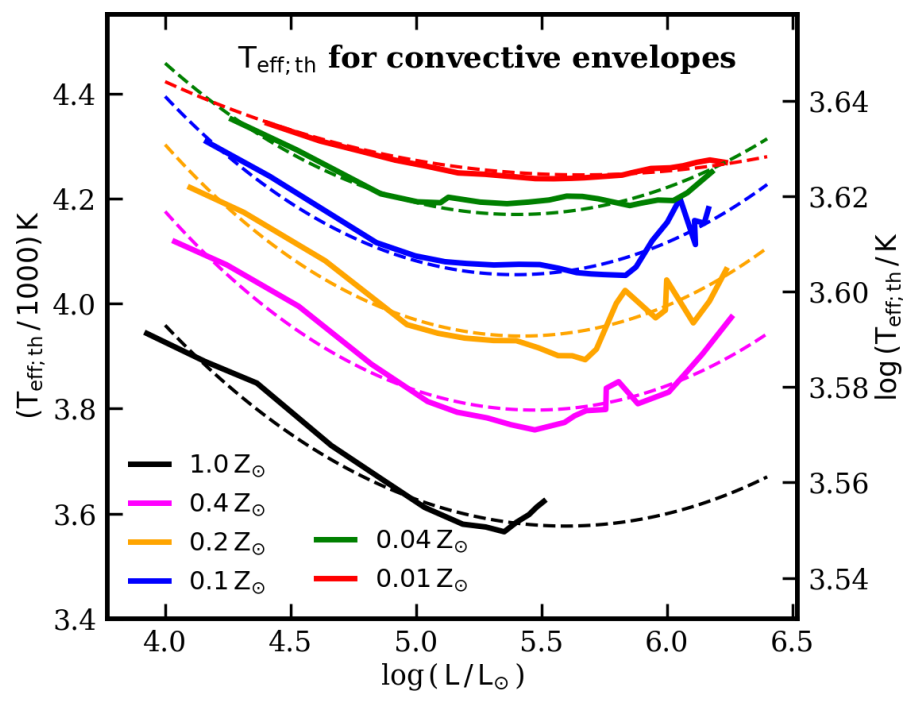

Fig. 6: Threshold effective temperature $T_{\text {eff;th }}$ below which at least $10 \%$ of the outer mass in a star is convective. Solid lines correspond to our stellar models, and the dashes lines show the numerical fit described with Eqs. 1 and 2.

about the type of the stellar envelope. For this reason, stability criteria are sometimes based on the evolutionary type of the star alone (e.g., Belczynski et al. 2008; Vigna-Gómez et al. 2018). This can lead to large errors: as Fig. 3 shows, the occurrence of outer convective envelopes cannot be unambiguously linked to any particular evolutionary phase. In particular, the mass transfer instability (and CE evolution) may be substantially overpredicted in this calculation if $\mathrm{CHeB}$ donors are assumed to respond to mass loss as convective stars.
Instead, mass transfer stability criteria should include a relation to the effective temperature of the donor star, which is the primary factor that determines the occurrence of a convective envelope. In Fig. 6 we plot as a function of luminosity and for various metallicities the threshold effective temperature $T_{\text {eff; }}$ below which at least $10 \%$ of the outer mass of the star is convective. Solid lines correspond to the stellar tracks computed in this work (see Fig. 1 and C.1 for HR diagrams). Dashed lines are the result of a numerical fit described by the following relation:

$\log \left(T_{\text {eff; th }} / \mathrm{K}\right)=a_{1} \log \left(L / L_{\odot}\right)^{2}+a_{2} \log \left(L / L_{\odot}\right)+a_{3}$,

where the coefficients $a_{i}$ for $i$ in $\{1,2,3\}$ are given as a function of metallicity,

$a_{i}=b_{1 i} \log \left(Z / Z_{\odot}\right)^{2}+b_{2 i} \log \left(Z / Z_{\odot}\right)+b_{3 i}$.

The values of the nine $b_{i j}$ coefficients are given in Table 1 . Notably, $T_{\text {eff; th }}$ in Fig. 6 depends on $Z$ : the lower the metallicity, the higher the threshold temperature for convective envelopes. The reason is that the Hayashi line depends on metallicity for a given value of the mixing-length parameter, where fully convective stars of high $Z$ have larger radii (and lower effective temperatures) than their lower metallicity counterparts. It should be noted, however, that the debate on whether the effective temperatures of RSGs (an indication for the Hayashi line) do indeed depend on their metallicity is still ongoing. Based on effective temperatures derived from spectral energy distribution fits, Davies et al. (2015) and Patrick et al. (2015) did not find any significant trend between $Z$ and $T_{\text {eff }}$ of RSGs of various metallicities from several different host galaxies (although this conclusion may depend on the method for determining $T_{\text {eff }}$, see Sec. 4.5 of Chun et al. 2018). Britavskiy et al. (2019) performed a similar analysis on a sample of RSGs from dwarf irregular galaxies from the Local Group, arguing in favor of a trend in which the minimum effective temperature increases toward lower metallicities. 
Table 1: Values of $b_{i j}$ coefficients in Eq. 2.

\begin{tabular}{c|ccc}
\hline$b_{i j}$ & $i=1$ & $i=2$ & $i=3$ \\
\hline$j=1$ & -0.0060 & -0.0066 & 0.0173 \\
$j=2$ & 0.0596 & 0.0587 & -0.1940 \\
$j=3$ & -0.1637 & -0.1967 & 4.0962 \\
\hline
\end{tabular}

Another piece of the puzzle has recently been added by Chun et al. (2018), who found that the inferred effective temperatures of RSGs in various local galaxies could be better matched by stellar models in which the mixing length increases with metallicity (see also Tayar et al. 2017). With all these results in mind, we stress that our fits in Fig. 6 are primarily meant to serve as a simple and approximate method for determining the transition from radiative to convective-envelope giants in population synthesis. We especially urge caution when a connection is made between the metallicity trend in Fig. 6 and expected effective temperatures of RSGs or maximum stellar radii of stars at different metallicities.

\section{Comparison with observations: clues for post-MS expansion}

The question of the post-MS expansion and blue-to-red evolution of massive stars is a long-standing problem in stellar astrophysics, with models being very sensitive to the adopted assumptions (see e.g., Langer 1991; Eldridge et al. 2008; Meynet et al. 2013). Most notably, it was shown that the degree of the HG expansion crucially depends on the efficiency of overshooting and semiconvective mixing (Langer et al. 1989; Stothers \& Chin 1992; Langer \& Maeder 1995), with rotational mixing also playing a role, see Georgy et al. (2013). The observable BSGto-RSG ratio can be used as a diagnostic tool in an attempt to calibrate the models (e.g., Eldridge et al. 2008; Georgy et al. 2013; Choi et al. 2016). Traditionally, the BSG group comprises $\mathrm{O}, \mathrm{B}$, and A stellar types $\left(\log T_{\text {eff }} \gtrsim 3.9\right)$ and the RSG group is composed of $\mathrm{K}$ and $\mathrm{M}$ supergiants $\left(\log T_{\text {eff }} \lesssim 3.7\right.$, e.g., Eggenberger et al. 2002; Massey \& Olsen 2003). In this way, the BSG group includes MS stars (O-type stars) and is dominated by them in terms of numbers. This limits the accuracy of the method in constraining specifically the post-MS phase of evolution.

A slightly modified approach has recently been carried out by Schootemeijer et al. (2019), who constructed a sample of BSGs from the SMC by combining only the B- and A-type stars $\left(3.85 \gtrsim \log T_{\text {eff }} \lesssim 4.4\right)$. In this way, they were able to probe predominantly the post-MS phase of evolution at the SMC metallicity (see Fig. 1). Notably, Schootemeijer et al. (2019) showed that models that assume the Schwarzschild criterion for convection (an assumption that is often made in previous calculations of massive-star models), which is equivalent to extremely efficient semiconvection, tend to underestimate the degree of the post-MS expansion and underpredict the number of RSGs. The disagreement of such models with observations has been pointed out by previous authors (e.g., Georgy et al. 2013).

\subsection{SMC-like metallicity $\left(Z=0.2 Z_{\odot}\right)$}

We took an approach similar to that of Schootemeijer et al. (2019) and compared our $Z=0.2 Z_{\odot}$ models with the observed population of luminous $\left(\log \left(L / L_{\odot}\right) \geq 4\right.$.7) supergiants in the SMC with effective temperatures $\log \left(T_{\text {eff }} / \mathrm{K}\right) \lesssim 4.35$. Following Drout et al. (2009), we defined RSGs as supergiants cooler than a threshold effective temperature $\log \left(T_{\text {eff;thresh }} / \mathrm{K}\right)=3.68$.
We aim to compare the number of RSGs to the number of hotter supergiants from the effective temperature range between $\log \left(T_{\text {eff }} / \mathrm{K}\right)=3.68$ and 4.35 . In this way, we constrain the postMS expansion of our models, which either expand all the way to the red giant branch during the $\mathrm{HG}$ phase and spend most of the $\mathrm{CHeB}$ lifetime as RSGs, or they complete the HG expansion as hotter stars and spend a significant fraction of the $\mathrm{CHeB}$ lifetime with $\log \left(T_{\text {eff }} / \mathrm{K}\right)$ between 3.68 and 4.35 . We note that in our $Z=0.2 Z_{\odot}$ tracks the convective RSG branch is located at slightly lower temperatures (at $\log \left(T_{\text {eff }} / \mathrm{K}\right) \lesssim 3.6$, depending on luminosity, see Fig. 1) than the assumed threshold temperature $\log \left(T_{\text {eff; thresh }} / \mathrm{K}\right)=3.68$ and than the measured effective temperatures of most of the RSGs in the SMC (e.g., obtained through spectral energy distrigution fitting Davies et al. 2018). This is a likely sign that our assumed mixing length parameter $\alpha=1.5$ is somewhat too small. For example, Chun et al. (2018) reported that models with $\alpha \approx 2$ agree better with temperatures of cool supergiants from the SMC. However, this discrepancy in temperatures of RSGs does not significantly affect the prediction for the number of RSGs from our models because for the majority of the models, the time that stars spend with $\log \left(T_{\text {eff }} / \mathrm{K}\right)$ between 3.6 and 3.68 is far shorter than the $\mathrm{CHeB}$ lifetime. We point out that other factors such as the effect of binary interactions or the shortcomings of the mixing-length theory in massive convective-envelope giants (see App. A) are likely to be a higher-order source of uncertainty.

For the sample of RSGs, we relied on the most recent investigation of the population of cool supergiants in the SMC by Davies et al. (2018). The authors combined a number of input catalogs in order to construct a highly complete sample of cool luminous SMC stars with effective temperatures $\log \left(T_{\text {eff }} / \mathrm{K}\right) \lesssim$ 3.74 (based on the effective temperature scale of Tabernero et al. 2018). Out of 151 stars with $\log \left(L / L_{\odot}\right) \geq 4.7$ in their sample, 147 meet our RSG criteria $\left(\log \left(T_{\text {eff }} / \mathrm{K}\right)<3.68\right)$.

As explained above, we mean to compare the number of RSGs with stars in the temperature range $3.68<\log \left(T_{\text {eff }} / \mathrm{K}\right)<$ 4.35. This includes both yellow supergiants (YSGs) $(3.68 \geq$ $\log \left(T_{\text {eff }} / \mathrm{K}\right) \leq 3.88$ Drout et al. 2009) and BSGs with $\log \left(T_{\text {eff }} / \mathrm{K}\right)$ between 3.88 and 4.35. To construct a combined sample of YSGs and BSGs, we relied on several different studies. In the temperature range $3.68<\log \left(T_{\text {eff }} / \mathrm{K}\right)<3.74$, four YSGs were reported by Davies et al. (2018). A survey by Neugent et al. (2010), targeting YSGs and cool BSGs and identifying their SMC membership based on their radial velocities, found 40 stars in the temperature range $3.74<\log \left(T_{\text {eff }} / \mathrm{K}\right)<4.1$ (eight of which were YSGs at $\left.\log \left(T_{\text {eff }} / \mathrm{K}\right)<3.88\right)$. The authors estimated the completeness of their sample to be high and that only a few rather than tens of objects were missing, especially at the higher luminosity end that we are interested here (i.e., $\left.\log \left(L / L_{\odot}\right)>4.7\right)$. Finally, 42 BSGs in the SMC with temperatures within $4.1<\log \left(T_{\text {eff }} / \mathrm{K}\right)<4.35$ have recently been reported by Kalari et al. (2018) with luminosities that meet our criteria. In total, the authors provided physical parameters of 69 BSGs, which were selected from the OGLE-II I-band imagining survey based on availability of multi-epoch photometry. Notably, a total of 110 BSGs were identified in the OGLE-II I-band data, which in turn is a fraction of 179 SMC BSGs reported in the catalog of massive SMC stars by Bonanos et al. (2010).

The combined number of BSGs and YSGs in our sample is thus 86, and the ratio of BSGs plus YSGs to RSGs is equal to $86 / 147 \approx 0.59$. As described above, the number of luminous BSGs in our sample is likely underestimated in the temperature range $4.1<\log \left(T_{\text {eff }} / \mathrm{K}\right)<4.35$, possibly by a factor of a few. Nonetheless, we consider the samples of RSGs and BSGs plus 

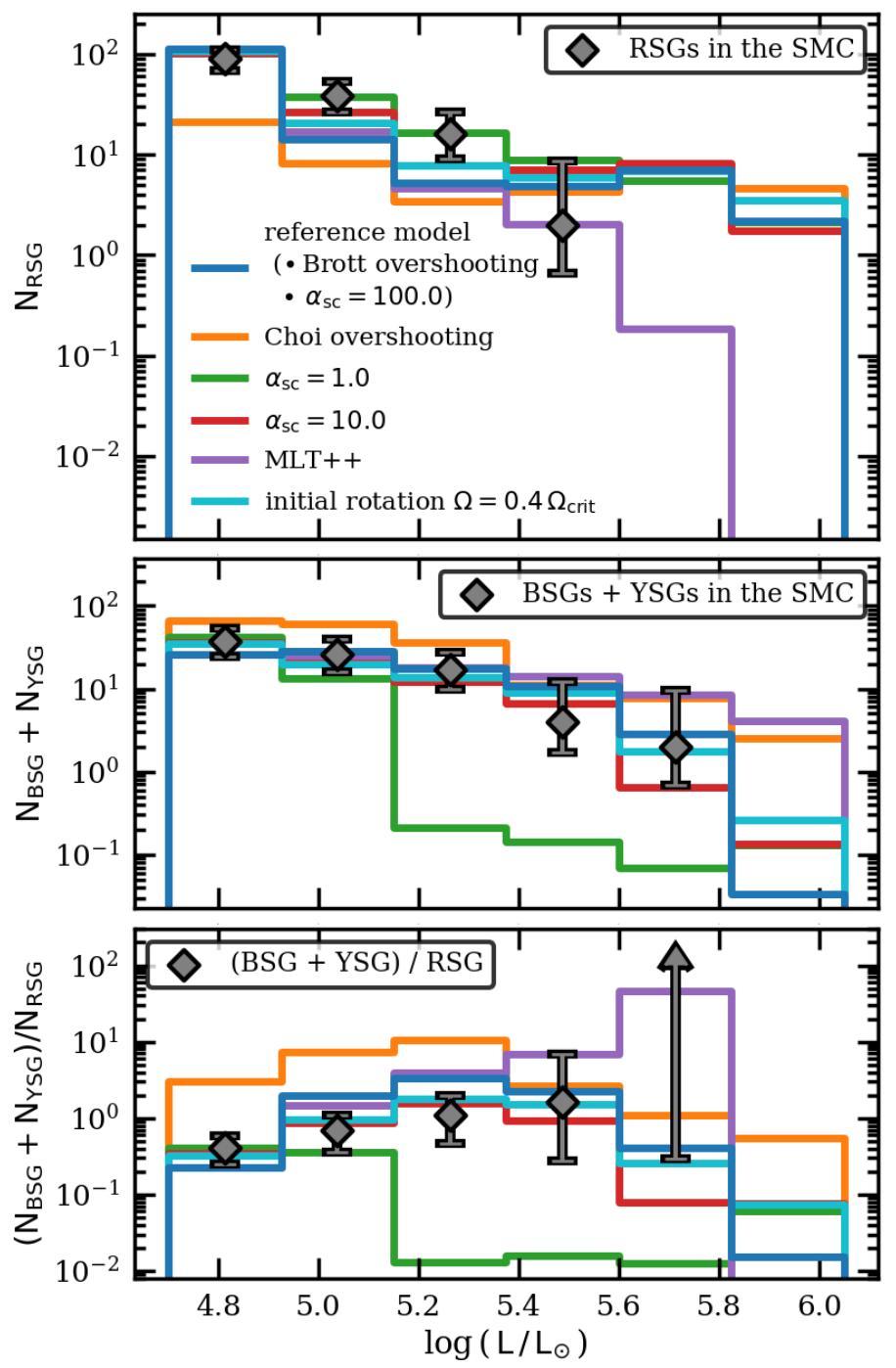

Fig. 7: Distributions of RSG and BSG plus YSG number counts (top and middle panels, respectively), as well as the number ratio of BSGs plus YSGs to RSGs (bottom panel) in logarithmic luminosity bins between 4.7 and 6.05 in $\log \left(L / L_{\odot}\right)$. Diamond scatter points correspond to observational samples of 147 RSGs (with $\log \left(T_{\text {eff }} / \mathrm{K}\right)<3.68$ ) and 86 BSGs plus YSGs (with $\log \left(T_{\text {eff }} / \mathrm{K}\right.$ ) between 3.68 and 4.35). Uncertainties correspond to $90 \%$ confidence limits assuming a Poissonian distribution for the number of supergiants in each bin. Only a lower limit is available for the ratio of BSGs plus YSGs to RSGs in the luminosity bin $5.6 \lesssim \log \left(L / L_{\odot}\right) \lesssim 5.8$. Solid-line histograms, normalized to 233 stars in total, correspond to several different sets of stellar tracks at $Z=0.0034=0.2 Z_{\odot}$ metallicity.

YSGs constructed in this way to be representative of the underlying population and sufficiently complete to compare with our models.

In Fig. 7 we plot luminosity distributions of RSGs and BSGs plus YSGs (top and middle panels, respectively), and the number ratio of BSGs plus YSGs to RSGs (bottom panel). Diamond points correspond to the observational samples described above, with Poissonian uncertainties marked as well $(90 \%$ confidence level). Solid-line histograms correspond to several different sets of stellar tracks at $Z=0.0034=0.2 Z_{\odot}$ (see Sec. 3.3 for a description of various models). To construct the histograms, we assumed a constant star formation rate and the Salpeter initial mass function slope for massive stars $\left(d N / d M \propto M^{-2.35}\right.$ Salpeter 1955). We normalized the histograms to the total number of 233 supergiants in the observed sample. We stress that the comparison in Fig. 7 is rather tentative because it attempts to compare single stellar tracks with a population of stars that are likely affected by binary evolution (most massive stars are born in binaries; Sana et al. 2012; Moe \& Di Stefano 2017). Additionally, the BSG, YSG, and RSG samples are not constructed in an unbiased and homogeneous way.

In the low-luminosity range $\left(\log \left(L / L_{\odot}\right) \lesssim 5.2\right)$, most of the models agree reasonably well with observations, up to a factor of a few in BSG plus YSG and RSG counts. Because the character of the comparison is tentative, we deem these discrepancies insufficient to confidently reject the models. The possible exception is the model with Choi overshooting, for which the ratio of BSGs plus YSGs to RSGs appears to be significantly too high in all three low luminosity bins $\left(\log \left(L / L_{\odot}\right)<5.4\right)$. It seems challenging to explain this discrepancy by the unaccounted-for binary effects because binary interactions would likely further reduce the theoretically expected number of RSGs (e.g., stars that lose mass in mass transfer become bluer). Unless the observed sample is missing most of the SMC BSGs or YSGs and the real ratio of BSGs plus YSGs to RSGs is a few times higher, the model with Choi overshooting is most likely disfavored.

In the high-luminosity range the discrepancies between models and observations become larger. In particular, no RSGs are observed in the SMC with luminosities above $\log \left(L / L_{\odot}\right) \approx$ 5.6 (the highest luminosity reported by Davies et al. 2018 is $\left.\log \left(L / L_{\odot}\right)=5.55 \pm 0.01\right)$, while all models but one predict at least a few of them in every 233 SMC supergiants. At the same time, several BSGs are observed with luminosities between 5.6 and 5.8 in $\log \left(L / L_{\odot}\right)$. This may indicate that stars with masses $\gtrsim 40 M_{\odot}$ at $Z=0.2 Z_{\odot}$ either do not expand to become RSGs at all or that the RSG phase is extremely short in this case. This might simply be due to rotation. For instance, Schootemeijer et al. (2019) found fewer RSGs in the upper part of the HR diagram in their models with rotation than in the nonrotating tracks (initial velocity of $300 \mathrm{~km} \mathrm{~s}^{-1}$, see their Fig. 11). A short-lived (and therefore difficult to observe) RSG phase of $M \gtrsim 40 M_{\odot}$ stars might be a result of increased mass-loss rates due to dustdriven winds (Chieffi \& Limongi 2013) or proximity to the Eddington limit (Chen et al. 2015).

Alternatively, the lack of RSGs above $\log \left(L / L_{\odot}\right) \approx 5.6$ may suggest that density inversions that would form in envelopes of such stars (in 1D models at least) either do not form at all because of an additional energy transport mechanism that prevents superadiabacity, or that such inversions are prone to instabilities and extensive mass loss (possibly the luminous blue variables phenomenon). For this reason, the model in which the formation of density inversions is prevented (the MLT++ approach) does not have solutions in the upper right corner of the HR diagram (see Fig. 5) and is statistically consistent with the lack of RSGs above $\log \left(L / L_{\odot}\right)=5.6$ in Fig. 7. On the other hand, the MLT++ model predicts about 15 BSGs above $\log \left(L / L_{\odot}\right)=5.6$, while only 2 such stars are present in the observed samples. Finally, the lack of RSGs from $M \gtrsim 40 M_{\odot}$ stars might arise because binary interactions dominate the evolution of massive stars and prevent them from becoming as large as $\gtrsim 2000 R_{\odot}$. Regardless of the reason, it seems unlikely that it has much to do with the tendency of some of the low-metallicity stars to begin the $\mathrm{CHeB}$ phase already as BSGs. We note that there appears to be a general lack of very massive (luminous; $\log \left(L / L_{\odot}\right)>5.8$ ) stars in the observed samples compared to our theoretical predictions. 
This might be an indication that very massive stars $\left(M \gtrsim 60 M_{\odot}\right)$ are rare in the SMC for reasons that are yet to be understood.

The middle panel of Fig. 7 shows that most of the models agree reasonably well with observations. The only exception are tracks that are computed with the lowest efficiency of semiconvective mixing, that is, $\alpha_{\mathrm{sc}}=1$. In this case, the model predicts no BSGs or YSGs with luminosities $\log \left(L / L_{\odot}\right)>5.2$ in the observed sample, while in reality, there are 23 such stars. Consequently, the ratio of BSGs plus YSGs to RSGs at $\log \left(L / L_{\odot}\right)>$ 5.2 in the model with $\alpha_{\mathrm{sc}}=1$ is strongly inconsistent with the observations (see the bottom panel).

One possible explanation for this discrepancy that should be considered is the effect of mass transfer evolution in binary systems. As we show in our upcoming paper, Klencki et al. (in prep), donor stars in stable mass transfer events are unlikely to help alleviate the issue because in the case of the $\alpha_{\mathrm{sc}}=1$ model, they would quickly become too hot to qualify for our BSG or YSG categories. On the other hand, sufficiently rejuvenated accretor stars or products of stellar mergers from early case B (or very late case $\mathrm{A}$ ) mass transfer might produce $\mathrm{CHeB}$ supergiants that evolve as BSGs or YSGs with luminosities $\log \left(L / L_{\odot}\right)>5.2$ (Justham et al. 2014). Because the parameter space for this type of evolution is limited, however, it is not clear whether such mass gainers or stellar mergers can fully account for the underabundance of BSGs plus YSGs from single stellar evolution in the model with $\alpha_{\mathrm{sc}}=1$ (although mass gainers in general may be quite common among apparently single stars; de Mink et al. 2014). With this caveat in mind, we consider the model with $\alpha_{\mathrm{sc}}=1$ (and similar models in which most of the CHeB evolution at $Z=0.2 Z_{\odot}$ takes place during the RSG stage) to be disfavored by the observations.

\subsection{Solar metallicity}

In the solar metallicity case, it is not feasible to use the ratio of BSGs plus YSGs to RSGs to constrain the post-MS radius evolution because of the larger (and more mass-dependent) sizes of MS stars. This makes it difficult to probe the post-MS phase of evolution alone. Additionally, it is more challenging to obtain a reasonably complete sample of supergiants in the Milky Way. Instead, we take a different approach to compare our $Z=Z_{\odot}$ tracks with observations. Recently, Castro et al. (2014) compiled a Galactic sample of massive stars in the spectroscopic HR diagram (sHR, Langer \& Kudritzki 2014) and compared them to single stellar tracks from Brott et al. (2011) and Ekström et al. (2012). Their sample is not complete; it was collected from various surveys that often had different scientific goals, therefore it is biased in several ways. This leads to an overpopulation of stars in certain regions such as the instability strip or the O- and early B-type temperature ranges. Nonetheless, certain sharp features of stellar tracks, such as the position of the TAMS or the red-giant branch, can be constrained.

In Fig. 8 we compare the sample collected by Castro et al. (2014) with our reference model in the sHR diagram (where $\mathcal{L}=T_{\text {eff }}^{4} / g$ ). The observations are plotted in the background as a grayscale density map (reproduced after Castro et al. 2014 with permission from the authors). We overplot contours of the theoretical distribution of stars obtained from our single stellar tracks at $Z=Z_{\odot}$ in color. The theoretical prediction was obtained under the assumption of the Salpeter initial mass function for $M \geq 10 M_{\odot}$ (Salpeter 1955). We also assumed that all such massive stars are formed in binary systems (e.g., Duchêne \& Kraus 2013; Moe \& Di Stefano 2017) and that their evolution can be approximated with our single tracks only until the even-

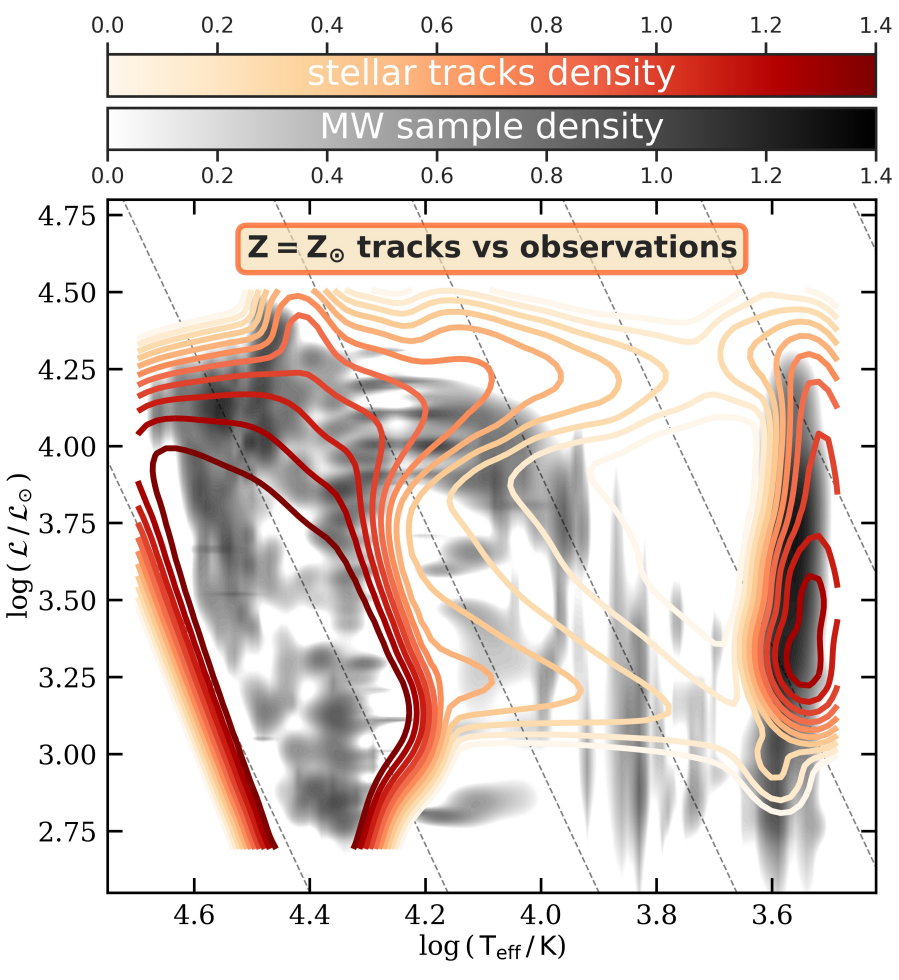

Fig. 8: Comparison of our stellar tracks computed at solar metallicity with the observed sample of Galactic massive stars collected by Castro et al. (2014). The observations are plotted as a density map in a logarithmic grayscale. We overplot contours of the theoretical distribution of stars obtained from our reference model at $Z=Z_{\odot}$ in color (also in a logarithmic scaling). The lack of observed stars in the parameter range of $3.7 \lesssim \log \left(T_{\text {eff }} / K\right) \lesssim 3.9$ and $3.5 \lesssim \log \left(\mathcal{L} / \mathcal{L}_{\odot}\right) \lesssim 4.0$ is a tentative piece of evidence that solar metallicity massive stars (mass range of about 16 to $40 M_{\odot}$ ) expand until the red giant branch during the $\mathrm{HG}$ phase.

tual RLOF. For the initial orbital period distribution, we assumed $d N / d(\log \mathrm{P}) \propto(\log \mathrm{P})^{-0.55}$ for $\log \mathrm{P}$ in range $[0.15,4.0]$ (Sana et al. 2012), and for simplicity, we assumed a fixed initial mass ratio of 0.5 and circular orbits. We assumed that when a star initiates RLOF and starts losing mass from its envelope, then it immediately becomes a hot helium star that does not fit into the $T_{\text {eff }}$ range in Fig. 8, and we did not include it in the theoretical distribution of stars in the figure. In our upcoming paper with binary models (Klencki et al. in prep.), we find that this is a good assumption in the case of stellar models in which the rapid HG expansion continues until the red giant branch (e.g., in the $Z=Z_{\odot}$ case), but not in the case of models with much smaller HG stars (e.g., the $Z=0.2 Z_{\odot}$ case). The $2 \mathrm{D}$ probability density function of stars in the sHR diagram was estimated from discrete stellar tracks computed in this study through a kernel-density estimation using Gaussian kernels (method stats.gaussian_kde from the SciPy package Virtanen et al. 2019).

The agreement of our theoretical distribution with observations is not perfect, although it is certainly not worse than in the case of the tracks by Brott et al. (2011) or Ekström et al. (2012), see Castro et al. (2014). Perhaps the most significant discrepancy is that we predict many more RSGs $\left(\log \left(T_{\text {eff }} / \mathrm{K}\right) \lesssim 3.68\right)$ and B-type stars $\left(\log \left(T_{\text {eff }} / \mathrm{K}\right)\right.$ between 4 and 4.2$)$ in the high- $\mathcal{L}$ region above 4.25 than what is observed. This might be an indication that the line-driven wind mass-loss prescriptions used 
in our models overestimate the mass-loss rates at solar metallicity (when a star loses mass from its envelope, it tends to move upward in the sHR diagram because $\mathcal{L} \propto L / M$ ). In the case of Btype massive stars, this is supported by some empirical evidence (e.g., Oskinova et al. 2011; Hainich et al. 2018).

From the point of view of the post-MS expansion and blue to red evolution, the interesting region of the sHR diagram is the parameter range of about $3.7 \lesssim \log \left(T_{\text {eff }} / K\right) \lesssim 3.9$ and $3.5 \lesssim \log \left(\mathcal{L} / \mathcal{L}_{\odot}\right) \lesssim 4.0$. This corresponds to the YSG position of massive stars in the range of about 16 to $40 M_{\odot}$. If, similarly to the subsolar metallicity case, these stars were to slow down their $\mathrm{HG}$ expansion and continue with the $\mathrm{CHeB}$ evolution as BSGs at first and later as YSGs, then we would expect them to populate this region in the sHR diagram. The lack of observed stars in this parameter range in the sample compiled by Castro et al. (2014) (which notably includes stars in the same $T_{\text {eff }}$ range, but at lower $\mathcal{L}$ ) is a tentative piece of evidence that solar metallicity massive stars expand to the red giant branch during the HG phase.

\section{Discussion}

The stellar tracks computed in this work show a gradual metallicity trend in the post-MS radius expansion of stars: the lower the metallicity, the stronger the tendency of massive stars to limit the rapid $\mathrm{HG}$ expansion and regain thermal equilibrium as heliumburning supergiants already in the blue part of the HR diagram, as much more compact stars than RSGs. In other words, the maximum size of massive HG stars decreases with metallicity. This trend has previously been described by Brunish \& Truran (1982) and Baraffe \& El Eid (1991) and also appears in some of the more recent single stellar tracks computed by other authors (e.g., Marigo et al. 2001; de Mink et al. 2008; Ekström et al. 2012; Georgy et al. 2013; Tang et al. 2014; Choi et al. 2016; Groh et al. 2019). In the following sections we discuss the origin and the robustness of this result, as well as some of its implications in the context of massive binary evolution.

\subsection{Why does the size of massive HG stars decrease with metallicity?}

Some low-metallicity models begin the $\mathrm{CHeB}$ phase as more compact stars than their higher metallicity counterparts because of the complicated interplay between at least two metallicitydependent factors: higher central temperatures and central densities at TAMS of low- $Z$ stars (resulting in a less pronounced mirror effect during the HG phase) and lower opacities in their envelopes (enabling smaller radius solutions) (see Brunish \& Truran (1982); Baraffe \& El Eid (1991); Langer (1991); Groh et al. (2019)). The wind mass-loss rate also plays a role. In general, a giant star with stronger winds has a more extended envelope than an otherwise very similar model with weaker winds, resulting in an increased rate of redward evolution (Brunish \& Truran 1982). A notable exception are the models of MS and WR stars with inflated envelopes, for which the inflated envelope solution was found to disappear for mass-loss rates above a certain critical value (Petrovic et al. 2006).

However, the way in which a star of a given mass and metallicity expands after the end of MS is not a robust result of evolutionary computations. In some cases, it depends sensitively on rather uncertain input parameters. For example, even at a metallicity as low as $Z=0.0017$, it is possible to have stellar tracks in which the most massive stars reach the RSG stage during the short-lived HG phase, similarly to solar metallicity models (see the model with $\alpha_{\mathrm{sc}}=1$ in Fig. 4).
The key factor that essentially determines the post-MS radius expansion in cases sensitive to model assumptions is the $\mathrm{H} / \mathrm{He}$ abundance profile in the layer just above the helium core. Depending on the efficiency of mixing, it can vary from a shallow linear slope (i.e., left at the end of the MS by the retreating convective core) to an extremely steep $\mathrm{H} / \mathrm{He}$ gradient, almost a step function (e.g., Schootemeijer \& Langer 2018). The abundance profile is important because it determines the location and strength of hydrogen-shell burning, which in turn determines the size of HG stars (see Kippenhahn \& Weigert 1990 and also Georgy et al. 2013). In Appendix B we show in detail how different efficiencies of semiconvective mixing $\left(\alpha_{\mathrm{sc}}=1\right.$ or 100) result in different $\mathrm{H} / \mathrm{He}$ abundance profiles above the helium cores of our $25 M_{\odot}$ models, leading to vastly different degrees of HG radius expansion (see also Schootemeijer et al. 2019).

In addition to semiconvection, other uncertain mixing processes affect the $\mathrm{H} / \mathrm{He}$ abundance in the layer above the helium core. For example, convective core overshooting (Langer et al. 1985; Stothers \& Chin 1992; Langer \& Maeder 1995), rotational mixing and instabilities (Georgy et al. 2013), and most likely, shell overshooting. Purely numerical aspects such as the determination of convective boundaries in stellar evolution codes also play a role (see, e.g., the convective pre-mixing approach in Paxton et al. 2018; 2019). The effect of all these factors on the abundance profile above the helium core is uncertain and most likely degenerate (Meynet et al. 2013)

\subsection{How robust is the trend with the metallicity?}

Because of the uncertainties in modeling the radial expansion of post-MS massive stars, it is vital to turn toward observations. The relative numbers of blue and yellow supergiants compared to red supergiants in the SMC indicate that already at $Z=Z_{\mathrm{SMC}} \approx 0.2 Z_{\odot}$, some massive stars (roughly between 15 and $40 M_{\odot}$ ) spend most of their CHeB lifetimes as BSGs or YSGs, see Sec. 4.1. The same conclusion has been reached previously by Schootemeijer et al. (2019). This allows us to reject models with relatively inefficient mixing in the zone above the helium core, for instance, the model with $\alpha_{\mathrm{sc}}=1.0$. On the other hand, the lack of YSGs with spectroscopic luminosities $\mathcal{L} / \mathcal{L}_{\odot} \gtrsim 3.6$ in the spectroscopic HR diagram of massive Galactic stars (Castro et al. 2014) indicates that stars of the same mass but higher metallicity (e.g., $Z \sim Z_{\odot}$ ) burn helium predominantly as RSGs, see Sec. 4.2.

These two pieces of evidence increase the credibility of our reference set of stellar tracks in terms of predicting the radius evolution of massive stars. Notably, the metallicity trend in the degree of post-MS expansion is present in all our models with variations in the input physical parameters as well as in the stellar tracks with rotation (see Sec. 3.3 and Fig. C.2 in the appendix). While the exact boundaries between evolutionary stages of the donor stars in interacting binaries in Fig. 3 should be considered rather uncertain, it appears that the trend with metallicity and its implications are a robust prediction of stellar models.

\subsection{WR stars formed through binary interactions}

As we illustrated in Sec. 3.2, the lower the metallicity, the more evolved are massive stars in binary systems at the point when they initiate mass transfer through RLOF. At $Z=Z_{\odot}$ and $0.4 Z_{\odot}$, a giant donor is almost always a star that has just completed its MS evolution and is rapidly expanding during the HG phase. During mass transfer, such a star very quickly $\left(\lesssim 10^{4} \mathrm{yr}\right.$, i.e., 
on a thermal timescale) loses almost its entire envelope and reveals its helium core (Kippenhahn \& Weigert 1967). In this way, a WR star can be formed by means of binary interactions (e.g., Maeder \& Meynet 1994; Bartzakos et al. 2001). The lack of a hydrogen envelope means that the helium core cannot grow in mass because of hydrogen-shell burning. Instead, the naked core loses mass in strong WR winds during the subsequent evolution. Recently, Woosley (2019) quantified these two effects on the final core masses and other core properties with respect to single stellar evolution. They did so by computing models of single naked helium stars, starting from the onset of helium burning. This choice of the starting point assumes that binary interactions strip the hydrogen envelope during the HG evolution (i.e., immediately before the onset of $\mathrm{CHeB}$ ), which is a well-justified assumption only in the high-metallicity case.

However, low-metallicity massive stars tend to remain more compact during the $\mathrm{HG}$ phase. As a result, at $Z \leq 0.2 Z_{\odot}$, the scenario in which the hydrogen envelope is lost during the $\mathrm{HG}$ evolution is no longer the dominating case. Instead, in somewhat wider systems (orbital periods $>10-50$ days, depending on metallicity), the RLOF mass transfer is initiated only after a significant fraction of the helium-burning lifetime, see Fig. 3. In such cases, the hydrogen envelope is preserved for longer and the helium core of the donor star has already grown in mass since the end of the MS as a result of hydrogen-shell burning. The remaining lifetime of such a donor after the start of RLOF is shorter than in the case of a HG donor star (in some cases, by more than an order of magnitude). This means that WR stars originating from intermediate- to long-period interacting binaries at low metallicity have much shorter lifetimes than stars in highermetallicity environments, which likely plays a role in the WR demographics in different host galaxies. Additionally, this shorter duration of the WR stage (after the RLOF) and the longer-lasting hydrogen-shell burning (before the RLOF and the resulting loss of the H-rich envelope) both work in favor of higher final core masses compared to stars that lose their hydrogen envelopes at the onset of CHeB (as assumed in Woosley 2019).

Interestingly, the time interval between the end of the MS and the onset of mass transfer through RLOF may affect the $\mathrm{H} / \mathrm{He}$ abundance gradient at the surface of the resulting WR star. The longer the envelope is preserved, the more time there is for internal mixing (mainly semiconvection) to steepen the $\mathrm{H} / \mathrm{He}$ gradient at the boundary of the helium core (see Appendix B and also Schootemeijer \& Langer 2018). These layers then become the surface layers of the WR star after the envelope is stripped. The surface $\mathrm{H} / \mathrm{He}$ abundance gradients of WR stars might be indirectly inferred from observations and used to constrain the evolution of their progenitors (Schootemeijer \& Langer 2018).

\subsection{Mass transfer evolution and slow envelope stripping}

As the metallicity decreases, increasingly more cases of mass transfer are initiated by slowly expanding CHeB giants (Fig. 3). Such donors do not necessarily become stripped of their entire envelopes on a short thermal timescale as is traditionally predicted for evolved giant donors (Kippenhahn \& Weigert 1967) and implemented in population-synthesis codes (e.g., Kruckow et al. 2018). Klencki \& Nelemans (2019) showed an example of a detailed model of an interacting binary system comprising a $25 M_{\odot}$ donor star (a $\mathrm{CHeB}$ giant) and a $10 M_{\odot} \mathrm{BH}$ accretor at subsolar metallicity $Z=0.2 Z_{\odot}$, in which mass transfer continues on a nuclear timescale of helium burning until the end of the $\mathrm{CHeB}$ phase. Throughout the entire mass transfer phase, the donor star maintains several solar masses of the hydrogen-rich envelope ( $\sim 2 M_{\odot}$ at the point of core-helium depletion). We discuss this type of mass transfer evolution in much more detail in Klencki et al. (in prep.).

When we assume that mass transfer from relatively compact $\mathrm{CHeB}$ stars could last for significantly longer than mass transfer from rapidly expanding $\mathrm{HG}$ donors (i.e., a nuclear timescale instead of a thermal timescale), then according to Fig. 3 we expect to find significantly more mass-transferring systems per unit of star formation rate in a low-metallicity galaxy compared to a high-metallicity one. This might be particularly interesting in the case of binaries with compact accretors, such as high-mass X-ray binaries. Observationally, X-ray binaries are indeed significantly more common in low-metallicity galaxies (e.g., by a factor of $\sim 10$ in blue compact dwarf galaxies; Brorby et al. 2014).

\subsection{Late cases of RLOF: progenitors of type II supernovae, long GRBs, and binary BH mergers?}

An interesting case is formed by systems in which the first RLOF takes place already very late into the evolution of the donor star, after most (or all) of the helium has been burned, for example, $Y_{\mathrm{C}}<0.05$. Such a case of mass transfer is sometimes referred to as case $\mathrm{C}$ mass transfer and corresponds to donors at the final stages of $\mathrm{CHeB}$ and to HeHG and SHeB donors in Figs. 3 and 4. We find that the parameter space for late case $C$ mass transfer is a strong function of metallicity. At $Z \gtrsim 0.2 Z_{\odot}$, such systems are very rare compared to all the other cases. At metallicities $Z \leq 0.1 Z_{\odot}$, the probability of late RLOF becomes significantly higher, up to $\sim 40 \%$ of all systems at $Z=0.01 Z_{\odot}$, see Fig. 3 .

In many systems that evolve through such a case of late mass transfer, only several thousand years are left from the point of RLOF to the collapse of the donor's core. This is not enough time to fully strip the hydrogen-rich envelope of a giant star, even if mass transfer is proceeding on a thermal timescale. Thus, a supernova from such a star would most likely appear as a hydrogen-rich type II supernova with a blue or yellow supergiant progenitor. In higher metallicity environments, a similar star would have been stripped of its hydrogen-rich envelope because mass transfer would have occurred far earlier during its evolution, and would explode as a type Ib or Ic supernova. Notably, any mass lost from a binary as a result of such a late case $\mathrm{C}$ mass transfer (stable or unstable) is expected to still be present in the proximity of the system at the time of supernova $(\sim 0.1 \mathrm{pc}$ for material ejected with velocity of $\sim 100 \mathrm{~km} \mathrm{~s}^{-1}$ traveling for $\sim 1000$ yr, see, e.g., Sun et al. 2020).

Systems in which late case $\mathrm{C}$ mass transfer becomes unstable and leads to a merger are among the promising candidates for the origin of long GRBs (Fryer et al. 2007). Because of the short remaining lifetime of the merger product, its $\mathrm{CO}$ core maintains rapid rotation until the collapse without losing much of the angular momentum in winds, as is required in the collapsar model (Woosley 1993). Notably, long GRBs are typically found in lowmetallicity hosts (Fruchter et al. 2006; Wolf \& Podsiadlowski 2007).

Evolution through unstable mass transfer and successful CE ejection is one of the most promising formation channels for compact binary mergers. Importantly, mass transfer is more prone to become unstable and to lead to $\mathrm{CE}$ evolution if the donor has a convective (rather than radiative) outer envelope (see Sec. 3.4 and references therein). Additionally, in the case of $\mathrm{BH}$ accretors, convective-envelope donors are more likely to satisfy the energy budget for the CE ejection (i.e., avoiding a merger within the CE; Kruckow et al. 2016). Here, we find that the parameter space for mass transfer from convective-envelope 
donors is very small at all metallicities, and that it disappears completely for $M \gtrsim 40 M_{\odot}$ donors at $Z \leq 0.04 Z_{\odot}$ metallicity. In reality, this parameter space may be even smaller than predicted in our models: the observational lack of RSGs above $\log \left(L / L_{\odot}\right) \approx 5.6$ in the nearby galaxies (Davies et al. 2018; Chun et al. 2018; , see also Sec. 4.1) may indicate that also in the higher metallicity environments $\left(Z \geq 0.2 Z_{\odot}\right)$ stars with masses above $\sim 40 M_{\odot}$ never develop outer convective envelopes. For the CE evolution scenario to explain the observed population of binary $\mathrm{BH}$ mergers, we either need this channel to work also when the mass transfer is initiated by a radiative envelope donor, or the parameters of progenitor binaries need to be fine-tuned to the small parameter space for mass transfer from convective envelope donors.

Notably, we find that almost all cases of mass transfer from convective envelope donors with masses $\lesssim 60 M_{\odot}$ and metallicities $\leq 0.2 Z_{\odot}$ are associated with the late case of RLOF from a core-helium depleted star, that is, a HeHG donor (see Fig. 3). The remaining lifetime of the donor star (up to $10^{4}$ years) is an upper limit for the duration of the subsequent BH-WR stage. This is at least an order of magnitude shorter than the full duration of the $\mathrm{CHeB}$ phase, that is, the lifetime of a WR star, if the envelope is stripped shortly after the end of the MS. The duration of the BH-WR stage is relevant for the degree of tidal spin-up of the WR star, which may be of importance for the spin of the second formed BH (e.g., Kushnir et al. 2016; Hotokezaka \& Piran 2017; Zaldarriaga et al. 2018).

\section{Conclusions}

We showed that metallicity has a strong influence on the type of mass transfer evolution that is expected in massive binaries. To do so, we computed a set of evolutionary tracks of stars between 10 and $80 M_{\odot}$ for six different metallicities ranging from $Z=0.017=Z_{\odot}$ to $Z=0.00017=0.01 Z_{\odot}$ (both nonrotating and rotating models with $\Omega_{\text {init }} / \Omega_{\text {crit }}=0.4$ ). We explored several variations of factors known to affect the radial expansion of massive stars (e.g., semiconvection and overshooting) and compared our models with observations. Our conclusions are summarized below.

- The lower the metallicity, the stronger the tendency of massive stars to remain relatively compact during the phase of rapid $\mathrm{HG}$ expansion $\left(\sim 100 R_{\odot}\right)$ and only reach the red giant branch towards the end of helium burning ( $\mathrm{CHeB}$ phase) or later, during contraction of the $\mathrm{CO}$ core (HeHG phase). At solar metallicity, no models behave in this way, and a postMS donor in an interacting binary is almost exclusively a rapidly expanding $\mathrm{HG}$ star. At $Z=0.1 Z_{\odot}$, stars in the mass range between $\sim 16$ and $50 M_{\odot}$ remain relatively compact before the onset of $\mathrm{CHeB}$, and a slowly expanding $\mathrm{CHeB}$ star is the typical donor in binaries with periods above $\sim 40$ days. At $Z=0.01 Z_{\odot}$, stars of all masses in our grid remain compact during the $\mathrm{HG}$ expansion. As a result, $\mathrm{CHeB}$ or HeHG stars are the most likely post-MS donors at very low metallicity.

- This metallicity trend is a relatively robust prediction of stellar models that is supported by the observations of blue and red supergiants in the Milky Way and in the SMC. The exact mass range for the compact $\mathrm{HG}$ evolution at any given metallicity is considered highly uncertain. Models that predict the $\mathrm{HG}$ expansion to reach the red giant branch for all masses at $Z=0.2 Z_{\odot}$ are ruled out by the observations (e.g., the model with a relatively inefficient semiconvection $\left.\alpha_{\mathrm{SC}}=1.0\right)$.
- At low metallicities $\left(\leq 0.2 Z_{\odot}\right)$, most massive stars can become stripped of their envelopes to form helium stars only after a substantial fraction of their helium-burning lifetime, as opposed to losing their envelopes shortly after the end of MS (as is often assumed, e.g., Woosley 2019). This implies a shorter duration of the WR phase and a more massive final core.

- As the metallicity decreases, increasingly more massive stars engage in their first mass transfer episode only very late in their evolution, after the core is almost completely depleted of helium $\left(Y_{\mathrm{C}}<0.05\right)$ and less then $10^{4} \mathrm{yr}$ remains until the collapse of the core. The short remaining lifetime is unlikely to be long enough to strip the giant star of its entire hydrogenrich envelope before the possible supernova.

- The binary parameter space for RLOF from convective envelope donors is very small, and it decreases with metallicity. For example, in the $0.1 Z_{\odot}$ metallicity case, it is only about 0.2 dex wide in $\log (P /$ day $)$, see Fig. 3. At metallicities $Z \leq 0.04 Z_{\odot}$, we find no mass transfer from convective envelope donors with masses above $\sim 40 M_{\odot}$ in our models. The lack of red supergiants with luminosities above $\log \left(L / L_{\odot}\right) \approx 5.6$ in local galaxies may indicate that the same is true for higher metallicities $Z \geq 0.2 Z_{\odot}$.

- No particular evolutionary stage can be used as a proxy for determining whether a star has a convective or a radiative envelope in population synthesis calculations. Instead, we provide fits for the threshold effective temperature below which stars develop outer convective envelopes (of at least $10 \%$ in mass), see Eqn. 1 and Fig. 6.

Acknowledgements. The authors would like to thank the referee for suggestions and comments that helped to improve the paper. We would also like to thank Selma de Mink, Stephen Justham, Abel Schootemeijer, Rob Farmer, Ylva Götberg, Martyna Chruslinska, Eva Laplace, and Manos Zapartas for valuable discussions and suggestions. We are grateful to Norberto Castro for sharing the density map of the sample of Galactic massive stars in the spectroscopic HR diagram. The authors acknowledge support from the Netherlands Organization for Scientific Research (NWO).

\section{References}

Asplund, M. 1998, A\&A, 330, 641

Baraffe, I. \& El Eid, M. F. 1991, A\&A, 245, 548

Bartzakos, P., Moffat, A. F. J., \& Niemela, V. S. 2001, MNRAS, 324, 18

Belczynski, K., Dominik, M., Bulik, T., et al. 2010, ApJ, 715, L138

Belczynski, K., Kalogera, V., Rasio, F. A., et al. 2008, ApJS, 174, 223

Böhm-Vitense, E. 1958, ZAp, 46, 108

Bonanos, A. Z., Lennon, D. J., Köhlinger, F., et al. 2010, AJ, 140, 416

Bressan, A., Fagotto, F., Bertelli, G., \& Chiosi, C. 1993, A\&AS, 100, 647

Britavskiy, N. E., Bonanos, A. Z., Herrero, A., et al. 2019, A\&A, 631, A95

Brorby, M., Kaaret, P., \& Prestwich, A. 2014, MNRAS, 441, 2346

Brott, I., de Mink, S. E., Cantiello, M., et al. 2011, A\&A, 530, A115

Brunish, W. M. \& Truran, J. W. 1982, ApJS, 49, 447

Cantiello, M., Langer, N., Brott, I., et al. 2009, A\&A, 499, 279

Castro, N., Fossati, L., Langer, N., et al. 2014, A\&A, 570, L13

Chen, Y., Bressan, A., Girardi, L., et al. 2015, MNRAS, 452, 1068

Chieffi, A. \& Limongi, M. 2013, ApJ, 764, 21

Choi, J., Dotter, A., Conroy, C., et al. 2016, ApJ, 823, 102

Chruslinska, M., Nelemans, G., \& Belczynski, K. 2019, MNRAS, 482, 5012 Chun, S.-H., Yoon, S.-C., Jung, M.-K., Kim, D. U., \& Kim, J. 2018, ApJ, 853,

Claret, A. \& Torres, G. 2018, ApJ, 859, 100

Davies, B., Crowther, P. A., \& Beasor, E. R. 2018, MNRAS, 478, 3138

Davies, B., Kudritzki, R.-P., Gazak, Z., et al. 2015, ApJ, 806, 21

de Mink, S. E., Cantiello, M., Langer, N., et al. 2009, A\&A, 497, 243

de Mink, S. E. \& Mandel, I. 2016, MNRAS, 460, 3545

de Mink, S. E., Pols, O. R., \& Yoon, S.-C. 2008, in American Institute of Physics

Conference Series, Vol. 990, First Stars III, ed. B. W. O'Shea \& A. Heger, 230-232

de Mink, S. E., Sana, H., Langer, N., Izzard, R. G., \& Schneider, F. R. N. 2014, ApJ, 782, 7 
Drout, M. R., Massey, P., Meynet, G., Tokarz, S., \& Caldwell, N. 2009, ApJ, 703,441

Duchêne, G. \& Kraus, A. 2013, ARA\&A, 51, 269

Eggenberger, P., Meynet, G., \& Maeder, A. 2002, A\&A, 386, 576

Ekström, S., Georgy, C., Eggenberger, P., et al. 2012, A\&A, 537, A146

Eldridge, J. J., Izzard, R. G., \& Tout, C. A. 2008, MNRAS, 384, 1109

Fagotto, F., Bressan, A., Bertelli, G., \& Chiosi, C. 1994, A\&AS, 105, 29

Farmer, R., Fields, C. E., Petermann, I., et al. 2016, ApJS, 227, 22

Farmer, R., Renzo, M., de Mink, S. E., Marchant, P., \& Justham, S. 2019, ApJ, 887,53

Fruchter, A. S., Levan, A. J., Strolger, L., et al. 2006, Nature, 441, 463 Fryer, C. L., Mazzali, P. A., Prochaska, J., et al. 2007, PASP, 119, 1211 Ge, H., Webbink, R. F., Chen, X., \& Han, Z. 2015, ApJ, 812, 40

Georgy, C., Ekström, S., Eggenberger, P., et al. 2013, A\&A, 558, A103

Giacobbo, N., Mapelli, M., \& Spera, M. 2018, MNRAS, 474, 2959

Glatzel, W. \& Kiriakidis, M. 1993, MNRAS, 263, 375

Gräfener, G. \& Hamann, W. R. 2008, A\&A, 482, 945

Gräfener, G., Owocki, S. P., \& Vink, J. S. 2012, A\&A, 538, A40

Gräfener, G., Vink, J. S., de Koter, A., \& Langer, N. 2011, A\&A, 535, A56

Grevesse, N., Noels, A., \& Sauval, A. J. 1996, in Astronomical Society of the Pacific Conference Series, Vol. 99, Cosmic Abundances, ed. S. S. Holt \& G. Sonneborn, 117

Grevesse, N. \& Sauval, A. J. 1998, Space Sci. Rev., 85, 161

Groh, J. H., Ekström, S., Georgy, C., et al. 2019, A\&A, 627, A24

Hainich, R., Oskinova, L. M., Shenar, T., et al. 2018, A\&A, 609, A94

Hamann, W. R., Koesterke, L., \& Wessolowski, U. 1995, A\&A, 299, 151

Heger, A., Fryer, C. L., Woosley, S. E., Langer, N., \& Hartmann, D. H. 2003, ApJ, 591, 288

Heger, A., Langer, N., \& Woosley, S. E. 2000, ApJ, 528, 368

Heger, A. \& Woosley, S. E. 2002, ApJ, 567, 532

Herwig, F. 2000, A\&A, 360, 952

Hjellming, M. S. \& Webbink, R. F. 1987, ApJ, 318, 794

Hotokezaka, K. \& Piran, T. 2017, ApJ, 842, 111

Humphreys, R. M. \& Davidson, K. 1979, ApJ, 232, 409

Humphreys, R. M. \& Davidson, K. 1994, PASP, 106, 1025

Hurley, J. R., Pols, O. R., \& Tout, C. A. 2000, MNRAS, 315, 543

Hurley, J. R., Tout, C. A., \& Pols, O. R. 2002, MNRAS, 329, 897

Ivanova, N., Justham, S., Chen, X., et al. 2013, A\&A Rev., 21, 59

Justham, S., Podsiadlowski, P., \& Vink, J. S. 2014, ApJ, 796, 121

Kalari, V. M., Vink, J. S., Dufton, P. L., \& Fraser, M. 2018, A\&A, 618, A17

Kato, M. 1985, PASJ, 37, 311

Kippenhahn, R. \& Weigert, A. 1967, ZAp, 65, 251

Kippenhahn, R. \& Weigert, A. 1990, Stellar Structure and Evolution

Klencki, J., Moe, M., Gladysz, W., et al. 2018, A\&A, 619, A77

Klencki, J. \& Nelemans, G. 2019, in IAU Symposium, Vol. 346, IAU Symposium, ed. L. M. Oskinova, E. Bozzo, T. Bulik, \& D. R. Gies, 417-425

Kruckow, M. U., Tauris, T. M., Langer, N., Kramer, M., \& Izzard, R. G. 2018, MNRAS, 481, 1908

Kruckow, M. U., Tauris, T. M., Langer, N., et al. 2016, A\&A, 596, A58

Kushnir, D., Zaldarriaga, M., Kollmeier, J. A., \& Waldman, R. 2016, MNRAS, 462,844

Langer, N. 1991, A\&A, 252, 669

Langer, N. 2012, ARA\&A, 50, 107

Langer, N., El Eid, M. F., \& Baraffe, I. 1989, A\&A, 224, L17

Langer, N., El Eid, M. F., \& Fricke, K. J. 1985, A\&A, 145, 179

Langer, N., Fricke, K. J., \& Sugimoto, D. 1983, A\&A, 126, 207

Langer, N. \& Kudritzki, R. P. 2014, A\&A, 564, A52

Langer, N. \& Maeder, A. 1995, A\&A, 295, 685

Maeder, A. \& Meynet, G. 1987, A\&A, 182, 243

Maeder, A. \& Meynet, G. 1994, A\&A, 287, 803

Magic, Z., Serenelli, A., Weiss, A., \& Chaboyer, B. 2010, ApJ, 718, 1378

Mapelli, M., Colpi, M., \& Zampieri, L. 2009, MNRAS, 395, L71

Marchant, P., Langer, N., Podsiadlowski, P., et al. 2017, A\&A, 604, A55

Marigo, P., Girardi, L., Chiosi, C., \& Wood, P. R. 2001, A\&A, 371, 152

Massey, P. \& Olsen, K. A. G. 2003, AJ, 126, 2867

Meynet, G., Ekstrom, S., Maeder, A., et al. 2013, Models of Rotating Massive Stars: Impacts of Various Prescriptions, ed. M. Goupil, K. Belkacem, C. Neiner, F. Lignières, \& J. J. Green, Vol. 865, 3

Moe, M. \& Di Stefano, R. 2017, ApJS, 230, 15

Neugent, K. F., Massey, P., Skiff, B., et al. 2010, ApJ, 719, 1784

Nieuwenhuijzen, H. \& de Jager, C. 1990, A\&A, 231, 134

Oskinova, L. M., Todt, H., Ignace, R., et al. 2011, MNRAS, 416, 1456

Owocki, S. P., Gayley, K. G., \& Shaviv, N. J. 2004, ApJ, 616, 525

Patrick, L. R., Evans, C. J., Davies, B., et al. 2015, ApJ, 803, 14

Pavlovskii, K. \& Ivanova, N. 2015, MNRAS, 449, 4415

Paxton, B., Bildsten, L., Dotter, A., et al. 2011, ApJS, 192, 3

Paxton, B., Cantiello, M., Arras, P., et al. 2013, ApJS, 208, 4

Paxton, B., Marchant, P., Schwab, J., et al. 2015, ApJS, 220, 15

Paxton, B., Schwab, J., Bauer, E. B., et al. 2018, ApJS, 234, 34

Paxton, B., Smolec, R., Schwab, J., et al. 2019, ApJS, 243, 10
Petrovic, J., Pols, O., \& Langer, N. 2006, A\&A, 450, 219

Planck Collaboration, Ade, P. A. R., Aghanim, N., et al. 2016, A\&A, 594, A13 Puls, J., Kudritzki, R. P., Herrero, A., et al. 1996, A\&A, 305, 171

Puls, J., Vink, J. S., \& Najarro, F. 2008, A\&A Rev., 16, 209

Quataert, E., Fernández, R., Kasen, D., Klion, H., \& Paxton, B. 2016, MNRAS, 458,1214

Salpeter, E. E. 1955, ApJ, 121, 161

Sana, H., de Koter, A., de Mink, S. E., et al. 2013, A\&A, 550, A107

Sana, H., de Mink, S. E., de Koter, A., et al. 2012, Science, 337, 444

Sanyal, D., Grassitelli, L., Langer, N., \& Bestenlehner, J. M. 2015, A\&A, 580, A20

Sanyal, D., Langer, N., Szécsi, D., -C Yoon, S., \& Grassitelli, L. 2017, A\&A, 597, A71

Schootemeijer, A. \& Langer, N. 2018, A\&A, 611, A75

Schootemeijer, A., Langer, N., Grin, N. J., \& Wang, C. 2019, A\&A, 625, A132

Smith, N. 2014, ARA\&A, 52, 487

Soberman, G. E., Phinney, E. S., \& van den Heuvel, E. P. J. 1997, A\&A, 327, 620

Spera, M., Mapelli, M., \& Bressan, A. 2015, MNRAS, 451, 4086

Stothers, R. \& Chin, C.-W. 1973, ApJ, 179, 555

Stothers, R. B. \& Chin, C.-W. 1992, ApJ, 390, 136

Sun, N.-C., Maund, J. R., \& Crowther, P. A. 2020, arXiv e-prints, arXiv:2003.09325

Tabernero, H. M., Dorda, R., Negueruela, I., \& González-Fernández, C. 2018, MNRAS, 476, 3106

Tang, J., Bressan, A., Rosenfield, P., et al. 2014, MNRAS, 445, 4287

Tayar, J., Somers, G., Pinsonneault, M. H., et al. 2017, ApJ, 840, 17

Ulmer, A. \& Fitzpatrick, E. L. 1998, ApJ, 504, 200

van Marle, A. J., Owocki, S. P., \& Shaviv, N. J. 2008, MNRAS, 389, 1353

Vigna-Gómez, A., Neijssel, C. J., Stevenson, S., et al. 2018, MNRAS, 481, 4009

Vink, J. S. \& de Koter, A. 2005, A\&A, 442, 587

Vink, J. S., de Koter, A., \& Lamers, H. J. G. L. M. 2000, A\&A, 362, 295

Vink, J. S., de Koter, A., \& Lamers, H. J. G. L. M. 2001, A\&A, 369, 574

Vink, J. S., Muijres, L. E., Anthonisse, B., et al. 2011, A\&A, 531, A132

Virtanen, P., Gommers, R., Oliphant, T. E., et al. 2019, arXiv e-prints, arXiv:1907.10121

Webbink, R. F. 1984, ApJ, 277, 355

Wolf, C. \& Podsiadlowski, P. 2007, MNRAS, 375, 1049

Woods, T. E. \& Ivanova, N. 2011, ApJ, 739, L48

Woosley, S. E. 1993, ApJ, 405, 273

Woosley, S. E. 2019, ApJ, 878, 49

Yoon, S. C., Langer, N., \& Norman, C. 2006, A\&A, 460, 199

Yusof, N., Hirschi, R., Meynet, G., et al. 2013, MNRAS, 433, 1114

Zaldarriaga, M., Kushnir, D., \& Kollmeier, J. A. 2018, MNRAS, 473, 4174

Zampieri, L. \& Roberts, T. P. 2009, MNRAS, 400, 677 


\section{Appendix A: Density inversions, MLT++, and the Humphreys-Davidson limit}

In massive stars, subsurface convective zones can develop as a consequence of iron and helium opacity peaks (Cantiello et al. 2009). At the same time, the low density and temperature in the outermost envelope layers of extended supergiants makes the convective energy transport ineffective. This leads to superadiabatic temperature gradients and requires most of the energy to be transported through radiation (e.g., Pavlovskii \& Ivanova 2015). In the case of stars that evolve near their Eddington limit, the subsurface opacity peaks and inefficient convection lead to a situation in which the radiative luminosity locally exceeds the Eddington limit in the envelope. The structure can become stabilized by a density inversion (Gräfener et al. 2012). In this way, the formation of density inversions in 1D stellar models is a natural consequence of sufficiently high luminosities in massive stars, with the metallicity and opacities playing an important role (see also Paxton et al. 2013).

On the one hand, density inversions and inefficient superadiabatic convection pose a numerical challenge that can lead to prohibitively short time steps. At the same time, many authors have considered such inversions unphysical in the first place and applied ad hoc solutions in order to remove them from stellar models, for example, by substituting pressure scale height with density scale height (e.g., Stothers \& Chin 1973; Ekström et al. 2012; Yusof et al. 2013) or by capping the temperature gradient at an imposed upper limit (e.g., Bressan et al. 1993; Fagotto et al. 1994). Others would argue in favor of stability of density inversions (Glatzel \& Kiriakidis 1993; Sanyal et al. 2015; 2017) with the caveat that this inversion might be suppressed by extremely strong winds (Asplund 1998). In any case, the fact that in superadiabatic layers the convective velocity approaches the speed of sound indicates that the standard mixing-length theory is beyond its domain of applicability, and that most likely 3D hydrodynamical simulations are required in order to advance the ongoing debate. Similarly to other ad hoc solutions mentioned above, MLT ++ is a stellar engineering solution to prevent the formation of density inversions in MESA. It gradually reduces the temperature gradient and thus the superadiabaticy in some radiation-dominated convective zones (see Paxton et al. 2013), which not only eliminates density inversions, but also alleviates many numerical difficulties. Crucially, MLT++ also increases the effective temperature of the model, similarly to other methods of preventing density inversions (e.g., using the density scale height or increasing the mass-loss rate Maeder \& Meynet 1987). The increase in effective temperature, even though somewhat artificial in the ad hoc methods such as MLT ++ , mimics an effect that an increased mass-loss rate would have on stars in the upper right corner of the HR diagram. It has been proposed that exceeding the Eddington limit in subsurface layers, the associated density inversions, and hydrodynamical turbulence and shocks in superadiabatic convective zones might be responsible for extreme mass loss of luminous supergiants and the luminous blue variable phenomenon (e.g., Owocki et al. 2004; van Marle et al. 2008; Quataert et al. 2016). This finds support in the fact that the location of the Eddington limit in the HR diagram coincides with the empirical HD limit (Humphreys \& Davidson 1979; 1994; Ulmer \& Fitzpatrick 1998), a line in the upper right side of the HR diagram beyond which almost no stars are observed in the Milky Way and in the Large Magellanic Cloud. Moreover, the Eddington factor $\Gamma$ has been identified as the key parameter determining the mass-loss rates of massive WR stars (Vink et al. 2011; Gräfener et al. 2011). Chen et al. (2015) assumed that the same $\Gamma$ dependence of the mass-loss rates applies to all stars (together with the metallicity scaling proposed by Gräfener \& Hamann 2008), and obtained stellar tracks that agree reasonably well with the HD limit at solar and LMC metallicities. A similar effect could be achieved through the use of MLT++ or the density scale height in place of the pressure scale height in superadiabatic zones. In this sense, stellar tracks computed with MLT++ might be more accurate in estimating the binary parameter space for mass transfer from the most massive and largest stars in our grid ( $\left.\gtrsim 50 M_{\odot}\right)$. On the other hand, it is currently unknown whether the empirical HD limit also appears in low-metallicity environments $\left(Z \lesssim 0.2 Z_{\odot}\right)$. In the case of solar metallicity, for which the HD limit is best evidenced, our nonMLT++ tracks also reproduce the lack of stars in the upper right corner of the HR diagram. Additionally, Chun et al. (2018) found a better agreement with the Galactic sample of luminous RSGs when MLT++ was not used. It remains certain that any numerical results for the maximum radii of massive stars $\left(\gtrsim 50 M_{\odot}\right)$ based on 1D stellar evolution computations should be considered highly uncertain.

\section{Appendix B: Internal mixing during the Hertzprung gap phase}

During the MS evolution of a massive star, the shrinking convective core leaves a region with a composition gradient behind. The corresponding gradient of mean-molecular weight stabilizes this region against convection, and at least initially, the composition is primarily being mixed by a slower process: semiconvection (Langer et al. 1985). The semiconvective zone becomes most extended immediately after the end of the MS when the star rapidly expands during the HG phase and hydrogen-shell burning becomes important. The mixing that occurs during this short-lived phase $\left(\sim 10^{4} \mathrm{yr}\right)$ has a key effect on the stellar radius at the onset of helium burning and the blue to red evolution in the HR diagram (e.g., Langer 1991).

In Fig. B. 1 we show Kippenhahn diagrams of three models of a single $25 M_{\odot}$ star: at $0.2 Z_{\odot}$ with efficient semiconvection, at $0.2 Z_{\odot}$ but with less efficient semiconvection, and at $1.0 Z_{\odot}$ with efficient semiconvection. The diagrams are centered around the short-lived phase between the end of MS and the onset of helium burning, see also Fig. 5 of Schootemeijer et al. (2019). If semiconvection is efficient and quick to sufficiently flatten the composition gradient for convective mixing to fully kick in, then a single extended convective zone is eventually formed at the top of the hydrogen shell (the top and bottom panels). In the case of less efficient semiconvection, multiple separate convective zones are formed instead, with semiconvective regions in between them (see, e.g., Fig. 5 of Langer et al. 1985). This can be understood in the following way: with less efficient semiconvection, the composition gradient is not flatenned quickly enough for an extended convective zone to form during the short-lived HG phase. However, when any small zone becomes locally unstable to convection, its composition is mixed very quickly. This eliminates any composition gradient within that region, which helps to maintain convective mixing. At the same time, the gradient at the edges of the small convective region becomes steeper, which increases the stability of neighboring zones against convection, and they can only be mixed further by semiconvection. If semiconvection in such neighboring zones is not efficient, then the small convective zone becomes isolated and cannot merge with other similar small convective regions. An onion-like structure with multiple convective and semiconvective zones emerges, as in the middle panel of Fig. B.1. 


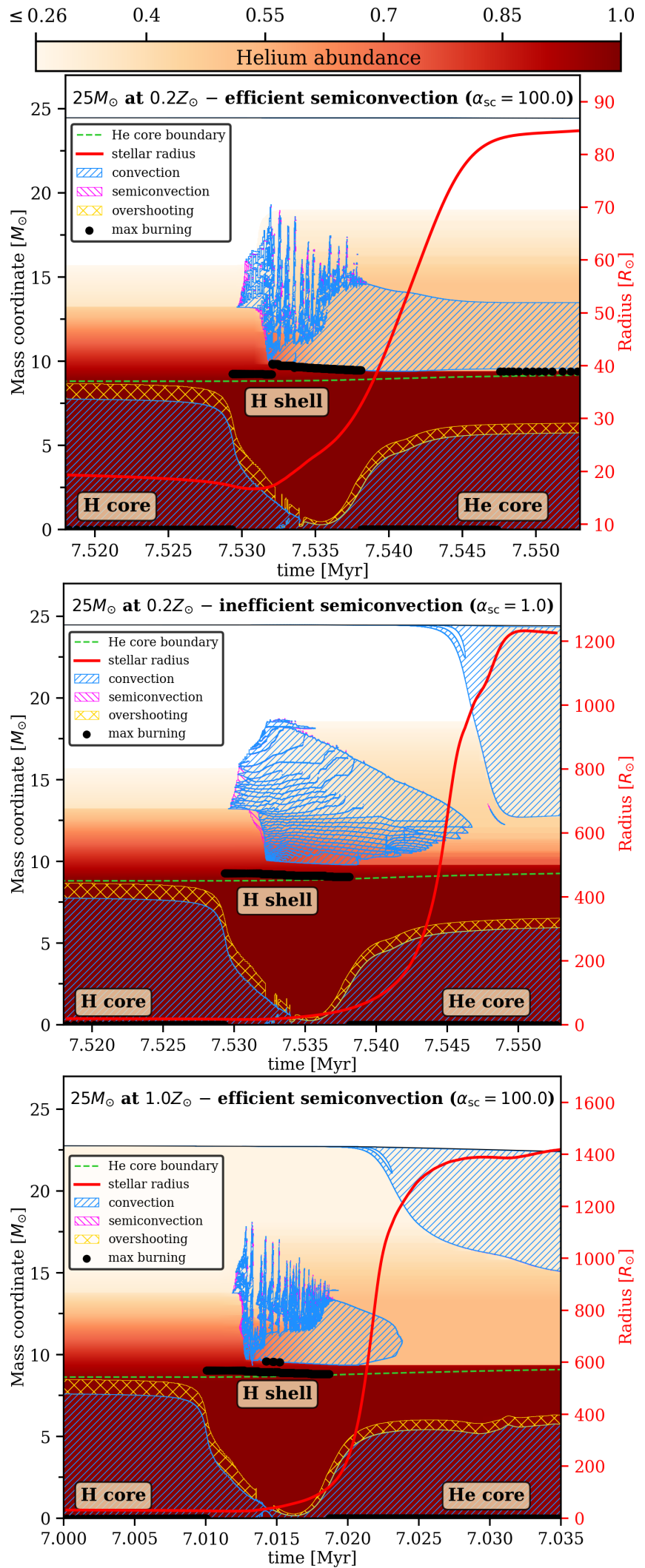

Fig. B.1: Kippenhahn diagrams of three models of a single $25 M_{\odot}$ star: at $0.2 Z_{\odot}$ with efficient semiconvection $\left(\alpha_{\mathrm{sc}}=100\right.$, top panel), at $0.2 Z_{\odot}$ but with inefficient semiconvection $\left(\alpha_{\mathrm{sc}}=\right.$ 1 , middle panel), and at $1.0 Z_{\odot}$ with efficient semiconvection $\left(\alpha_{\mathrm{sc}}=100\right.$, bottom panel). We also mark the stellar radius (red solid line, labeled on the right-hand side) to show the influence of mixing on the evolution in the HR diagram. In the solar metallicity case, even if the mixing is efficient, the model expands to $R>1000 R_{\odot}$ during the HG phase. The resulting helium abundance profiles are shown in Fig. B.2.

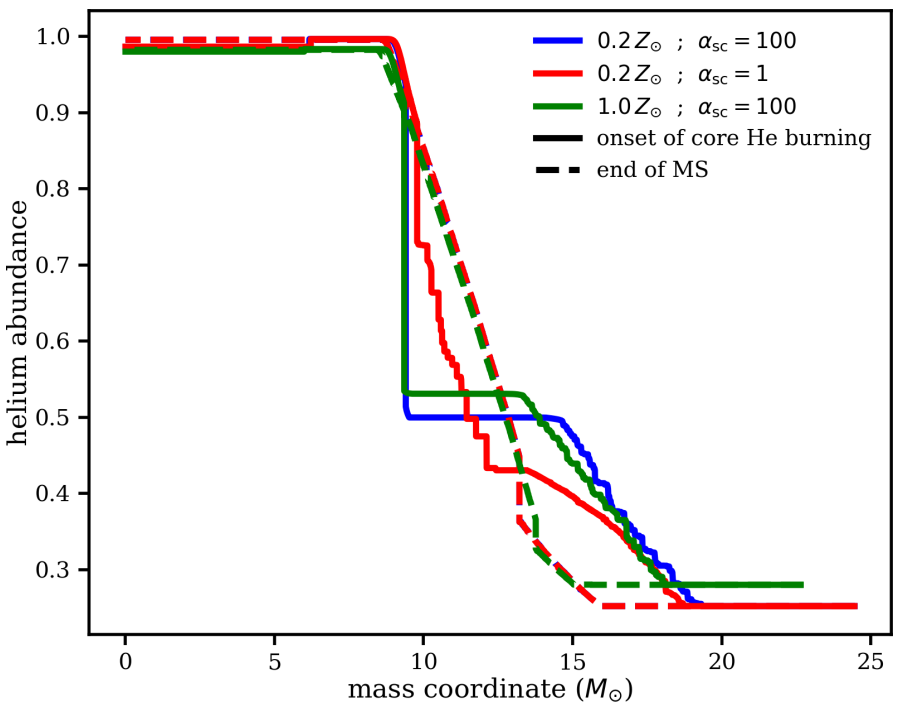

Fig. B.2: Helium abundance profiles of models shown in Fig. B.1, taken from the point at the end of the MS and before the mixing (dashed lines) and after a convective helium burning core fully develops (solid lines).

The interplay between semiconvective and convective mixing ultimately determines the abundance profile in the region above the hydrogen shell. In Fig. B.2 we show helium abundance profiles taken at the end of MS (before the mixing) and after the convective helium core develops (after the mixing) for the three models shown in Fig. B.1. Efficient mixing $\left(\alpha_{\mathrm{sc}}=100\right)$ results in a plateau of helium abundance at the bottom of the envelope, much different from the step-like profile that forms in the case of less efficient mixing ( $\left.\alpha_{\mathrm{sc}}=1\right)$. This plateau is a common feature of all our post-MS donor stars in binary models as well. See also Schootemeijer \& Langer (2018) for a discussion of how the resulting $\mathrm{H} / \mathrm{He}$ gradient is connected to surface abundances of WR stars. In the efficient mixing case, the envelope has become more enriched in helium, and at the same time, a larger amount of hydrogen has been brought down as fuel for the shell burning. In the solar metallicity case, even if the mixing is efficient, the model expands to $R>1000 R_{\odot}$ during the HG phase.

We comment that the forest-like structure of short-lived extended convective zones that form in models with efficient semiconvection has been found in MESA models by other author as well (e.g., Farmer et al. 2016; Schootemeijer \& Langer 2018). Its exact behavior appears very chaotic and is subject to numerical settings and resolution. This mixing affects the composition in the region above the single convective zone: it creates a steplike decrease in $Y$ between mass coordinates $\sim 15$ and $20 M_{\odot}$ in Fig. B.2, the details of which are considered highly uncertain. Notably, we have found that models running with convective premixing (Paxton et al. 2019) without any limits on the velocity of a convective boundary advance (CONV_PREMIX_TIME_FACTOR $=0.0$ in the MESA jargon) are effective in forming a single extended convective zone even with $\alpha_{\mathrm{sc}}=1$.

\section{Appendix C: Additional HR diagrams}

Article number, page 17 of 19 


\begin{tabular}{|llll|}
\hline$\circ$ & $\begin{array}{l}\text { post-MS position every 50,000 yr } \\
\text { end of core He burning }\end{array}$ & $\begin{array}{l}\text { onset of core C burning } \\
\text { outer conv envelope (>10\%) }\end{array}$ & $\diamond \begin{array}{l}\text { outer conv envelope (> 20\%) } \\
\text { TAMS }\end{array}$ \\
\hline
\end{tabular}
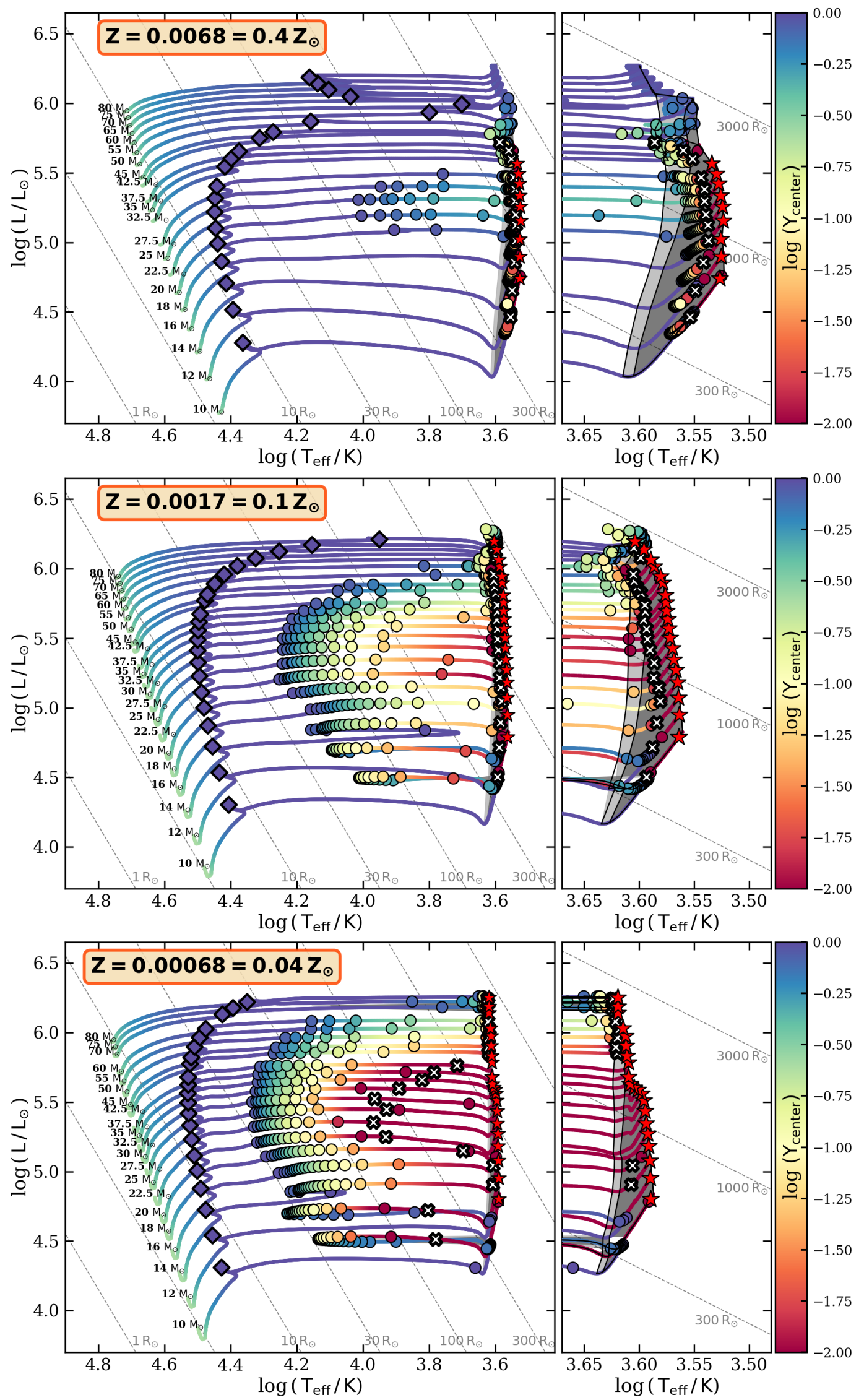

Fig. C.1: Same as Fig. 1 but for 3 additional metallicities. 

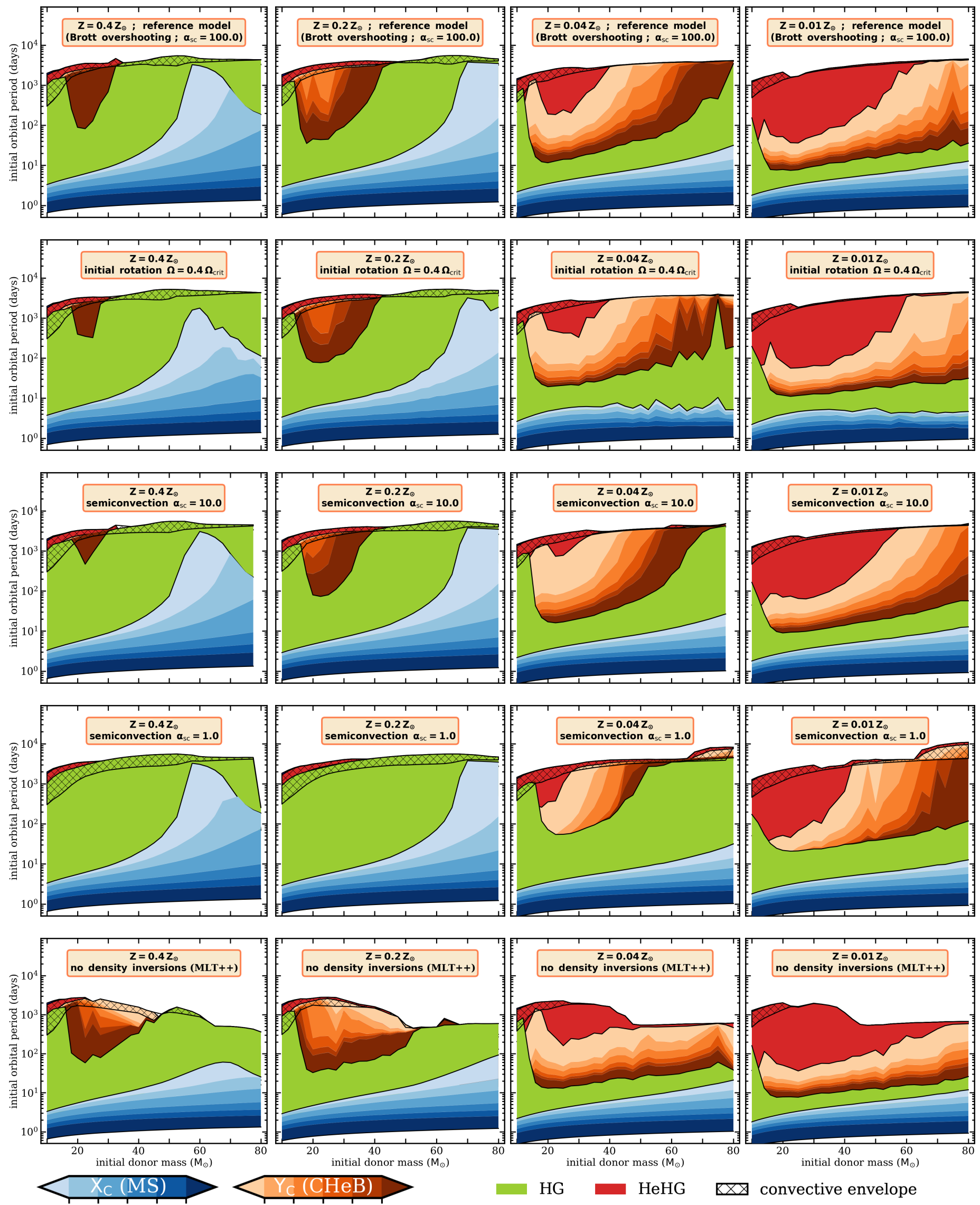

$\begin{array}{lllll}0.05 & 0.1 & 0.2 & 0.3 & 0.4\end{array}$

$\begin{array}{lllll}0.05 & 0.1 & 0.2 & 0.3 & 0.4\end{array}$

Fig. C.2: Same as Fig. 4, but for other metallicities. 